\title{
ON SOLVABILITY OF PERTURBED SOBOLEV TYPE EQUATIONS
}

\author{
V. E. FEDOROV AND O. A. RUZAKOVA
}

\begin{abstract}
Linear Sobolev type equations

$$
L \dot{u}(t)=M u(t)+N u(t), \quad t \in \overline{\mathbb{R}}_{+},
$$

are considered, with degenerate operator $L$, strongly $(L, p)$-radial operator $M$, and perturbing operator $N$. By using methods of perturbation theory for operator semigroups and the theory of degenerate semigroups, unique solvability conditions for the Cauchy problem and Showalter problem for such equations are deduced. The abstract results obtained are applied to the study of initial boundary value problems for a class of equations, the operators in which are polynomials of elliptic selfadjoint operators, including various equations of filtration theory. Perturbed linearized systems of the phase space equations and of the Navier-Stokes equations are also considered. In all cases the perturbed operators are integral or differential.
\end{abstract}

\section{$\S 1$. INTRODUCTION}

We consider the Cauchy problem

$$
u(0)=u_{0}
$$

for the Sobolev type equation

$$
L \dot{u}(t)=M u(t)+N u(t), \quad t \in \overline{\mathbb{R}}_{+} .
$$

This is an abstract form of initial boundary value problems for various equations and systems of equations modeling real processes [1, 2, 3, 4, Here $\mathfrak{U}$ and $\mathfrak{F}$ are Banach spaces, $L \in \mathcal{L}(\mathfrak{U} ; \mathfrak{F})$, i.e., $\mathcal{L}$ is a continuous linear operator, the operators $M, N$ belong to $\mathcal{C l}(\mathfrak{U} ; \mathfrak{F})$, i.e., they are linear, closed, and densely defined in $\mathfrak{U}$ and map $\mathfrak{U}$ to $\mathfrak{F}$. The papers [1, 3, 5, 6, 7, 8, are devoted to finding conditions that ensure the existence of resolving semigroups from several smoothness classes for the nonperturbed Sobolev type equation $(N=0)$. In particular, it was shown that in the case where ker $L \neq\{0\}$ such semigroups are degenerate. In other words, the identity of such a semigroup has a nontrivial kernel. If the operator $L^{-1} \in \mathcal{L}(F ; U)$ exists, then equation (1.2) can be reduced to the form

$$
\dot{u}(t)=L^{-1} M u(t)+L^{-1} N u(t), \quad t \in \overline{\mathbb{R}}_{+} .
$$

If the operator $L^{-1} M$ generates a $\left(C_{0}\right)$-continuous operator semigroup, then problem (1.1), (1.3) can be investigated by methods of perturbation theory for operator semigroups. The basis of that theory was established in the works [9, 10] by R. Phillips; see also the bibliography in [11] and Ivanov's papers [12, 13] concerning semigroup perturbations in locally convex spaces. Our aim in this paper is in applying the methods of perturbation theory for operator semigroups and of the theory of degenerate semigroups to the study of problem (1.1), (1.2) in the case where ker $L \neq\{0\}$. In this case the perturbed equation can be reduced to a system of two equations on mutually complementary subspaces, namely, the kernel and the image of the resolving semigroup for the

2000 Mathematics Subject Classification. Primary 34G25.

Key words and phrases. Perturbation theory, semigroup, Cauchy problem, Sobolev type equation. 
nonperturbed equation $(N=0)$. Since the equation on the image is resolved with respect to the derivative, the existence of its solution follows from Phillips' results concerning perturbed $\left(C_{0}\right)$-semigroups. The equation on the kernel contains a nilpotent operator applied to the derivative and cannot be analyzed in the general form. However, under some assumptions on the perturbed operator $N$, in this paper we find a solution of this equation and, thereby, of equation (1.2). We illustrate the abstract results obtained by examples of initial boundary value problems for partial differential equations and systems of equations.

\section{$\S 2$. Preliminaries}

Let $\mathfrak{U}, \mathfrak{F}$ be Banach spaces. The Banach space of all continuous linear operators from $\mathfrak{U}$ to $\mathfrak{F}$ will be denoted by $\mathcal{L}(\mathfrak{U} ; \mathfrak{F})$. The notation $\mathcal{L}(\mathfrak{U})$ will mean that $\mathfrak{F}=\mathfrak{U}$. The set of all closed linear operators with dense domain in a space $\mathfrak{U}$, with values in $\mathfrak{F}$, will be denoted by $\mathcal{C l}(\mathfrak{U} ; \mathfrak{F})$. The set $\mathcal{C l}(\mathfrak{U} ; \mathfrak{U})$ will be written as $\mathcal{C l}(\mathfrak{U})$.

Everywhere in what follows we assume that $L \in \mathcal{L}(\mathfrak{U} ; \mathfrak{F}), M \in \mathcal{C l}(\mathfrak{U} ; \mathfrak{F})$. We also denote $\rho^{L}(M)=\left\{\mu \in \mathbb{C}:(\mu L-M)^{-1} \in \mathcal{L}(\mathfrak{F} ; \mathfrak{U})\right\}, R_{\mu}^{L}(M)=(\mu L-M)^{-1} L, L_{\mu}^{L}(M)=$ $L(\mu L-M)^{-1}, R_{(\lambda, p)}^{L}(M)=\prod_{k=0}^{p} R_{\mu_{k}}^{L}(M), L_{(\lambda, p)}^{L}(M)=\prod_{k=0}^{p} L_{\mu_{k}}^{L}(M), \mathbb{N}_{0}=\mathbb{N} \cup\{0\}$, $\mathbb{R}_{+}=\{a \in \mathbb{R}: a>0\}$, and $\overline{\mathbb{R}}_{+}=\mathbb{R}_{+} \cup\{0\}$.

Definition 2.1. An operator $M$ is strongly $(L, p)$-radial if

(i) there exists $a \in \mathbb{R}$ such that $(a,+\infty) \subset \rho^{L}(M)$;

(ii) there exists $K>0$ such that for any $\mu_{k} \in(a,+\infty)$, any $k=0, \ldots, p$, and any $n \in \mathbb{N}$ we have

$$
\max \left\{\left\|\left(R_{(\mu, p)}^{L}(M)\right)^{n}\right\|_{\mathcal{L}(\mathfrak{U})},\left\|\left(L_{(\mu, p)}^{L}(M)\right)^{n}\right\|_{\mathcal{L}(\mathfrak{F})}\right\} \leq \frac{K}{\prod_{k=0}^{p}\left(\mu_{k}-a\right)^{n}} ;
$$

(iii) in $\mathfrak{F}$ there exists a dense subspace $\stackrel{\mathfrak{F}}{\mathrm{F}}$ such that

$$
\left\|M(\lambda L-M)^{-1} L_{(\mu, p)}^{L}(M) f\right\|_{\mathfrak{F}} \leq \frac{\operatorname{const}(f)}{(\lambda-a) \prod_{k=0}^{p}\left(\mu_{k}-a\right)}, \quad f \in \stackrel{\circ}{\mathfrak{F}}
$$

for all $\lambda, \mu_{0}, \mu_{1}, \ldots, \mu_{p} \in(a,+\infty)$;

(iv) for all $\lambda, \mu_{0}, \mu_{1}, \ldots, \mu_{p} \in(a,+\infty)$ we have

$$
\left\|R_{(\mu, p)}^{L}(M)(\lambda L-M)^{-1}\right\|_{\mathcal{L}(\mathfrak{F} ; \mathfrak{U})} \leq \frac{K}{(\lambda-a) \prod_{k=0}^{p}\left(\mu_{k}-a\right)} .
$$

We denote by $\mathfrak{U}^{0}\left(\mathfrak{F}^{0}\right)$ the kernel $\operatorname{ker} R_{(\mu, p)}^{L}(M)\left(\operatorname{ker} L_{(\mu, p)}^{L}(M)\right)$, and by $\mathfrak{U}^{1}\left(\mathfrak{F}^{1}\right)$ the closure of the subspace $\operatorname{im} R_{(\mu, p)}^{L}(M)\left(\operatorname{im} L_{(\mu, p)}^{L}(M)\right)$ in the space $\mathfrak{U}(\mathfrak{F})$. By $M_{k}\left(L_{k}\right)$ we denote the restriction of $M(L)$ to the subspace $\operatorname{dom} M_{k}=\mathfrak{U}^{k} \cap \operatorname{dom} M\left(\mathfrak{U}^{k}\right), k=0,1$.

Theorem 2.1 (6]). If $M$ is a strongly $(L, p)$-radial operator, then:

(i) $\mathfrak{U}=\mathfrak{U}^{0} \oplus \mathfrak{U}^{1}, \mathfrak{F}=\mathfrak{F}^{0} \oplus \mathfrak{F}^{1}$;

(ii) $L_{k} \in \mathcal{L}\left(\mathfrak{U}^{k} ; \mathfrak{F}^{k}\right), M_{k} \in \mathcal{C l}\left(\mathfrak{U}^{k} ; \mathfrak{F}^{k}\right), k=0,1$;

(iii) the operators $M_{0}^{-1} \in \mathcal{L}\left(\mathfrak{F}^{0} ; \mathfrak{U}^{0}\right)$ and $L_{1}^{-1} \in \mathcal{L}\left(\mathfrak{F}^{1} ; \mathfrak{U}^{1}\right)$ exist;

(iv) the operator $H=M_{0}^{-1} L_{0} \in \mathcal{L}\left(\mathfrak{U}^{0}\right)$ is nilpotent of degree at most $p$;

(v) there exists a strongly continuous resolving semigroup $\left\{U^{t} \in \mathcal{L}(\mathfrak{U}): t \in \overline{\mathbb{R}}_{+}\right\}$of the equation $L \dot{u}=M u$, and

$$
\left\|U^{t}\right\|_{\mathcal{L}(\mathfrak{U})} \leq K e^{a t}, \quad t \in \overline{\mathbb{R}}_{+} ;
$$

(vi) the infinitesimal generator of the $C_{0}$-continuous semigroup $\left\{U_{1}^{t}=\left.U^{t}\right|_{\mathfrak{U}^{1}} \in \mathcal{L}\left(\mathfrak{U}^{1}\right)\right.$ : $\left.t \in \overline{\mathbb{R}}_{+}\right\}$is the operator $L_{1}^{-1} M_{1} \in \mathcal{C l}\left(\mathfrak{U}^{1}\right)$. 
Remark 2.1. The projection along $\mathfrak{U}^{0}$ to $\mathfrak{U}^{1}$ (along $\mathfrak{F}^{0}$ to $\mathfrak{F}^{1}$ ) has the form

$$
P=\underset{\mu-\lim }{s \rightarrow+\infty}\left(\mu R_{\mu}^{L}(M)\right)^{p+1} \quad\left(Q=\underset{\mu \rightarrow+\infty}{s-\lim _{j}}\left(\mu L_{\mu}^{L}(M)\right)^{p+1}\right) .
$$

In the proof of (ii) we use the fact that, under the conditions of Theorem 2.1, we have $Q L=L P$ and $Q M u=M P u$ for $u \in \operatorname{dom} M$. Put $I-Q=Q_{0}$.

Theorem $2.2(\underline{6})$. Suppose $M$ is a strongly $(L, p)$-radial operator, and a function $f$ is such that $Q_{0} f \in C^{p+1}\left(\overline{\mathbb{R}}_{+} ; \mathfrak{F}\right), L_{1}^{-1} Q f \in C^{1}\left(\overline{\mathbb{R}}_{+} ; \mathfrak{F}\right)$. Then for any initial value

$$
u_{0} \in \mathcal{P}_{f}=\left\{u \in \operatorname{dom} M:(I-P) u=-\sum_{k=0}^{p} H^{k} M_{0}^{-1}\left(Q_{0} f\right)^{(k)}(0)\right\}
$$

there exists a unique solution $u \in C^{1}\left(\overline{\mathbb{R}}_{+} ; \mathfrak{U}\right) \cap C\left(\overline{\mathbb{R}}_{+} ; \operatorname{dom} M\right)$ of the Cauchy problem $u(0)=u_{0}$ for the equation $L \dot{u}=M u+f$. The solution has the form

$$
u(t)=U^{t} u_{0}+\int_{0}^{t} U^{t-s} L_{1}^{-1} Q f(s) d s-\sum_{k=0}^{p} H^{k} M_{0}^{-1}\left(Q_{0} f\right)^{(k)}(t) .
$$

Remark 2.2. We need the condition $u_{0} \in \operatorname{dom} M$ to ensure the differentiability of the function $v(t)=U^{t} u_{0}$. Strictly speaking, for this purpose the condition $u_{0} \in \mathfrak{U}^{0} \dot{+} \operatorname{dom} M_{1}$ would suffice.

Theorem 2.3 ([3]). Suppose $M$ is a strongly $(L, p)$-radial operator, and a function $f$ is such that $Q_{0} f \in C^{p+1}\left(\overline{\mathbb{R}}_{+} ; \mathfrak{F}\right), L_{1}^{-1} Q f \in C^{1}\left(\overline{\mathbb{R}}_{+} ; \mathfrak{F}\right)$. Then for any initial value $u_{0} \in \operatorname{dom} M$ there exists a unique solution $u \in C^{1}\left(\overline{\mathbb{R}}_{+} ; \mathfrak{U}\right) \cap C\left(\overline{\mathbb{R}}_{+} ; \operatorname{dom} M\right)$ of the problem $P u(0)=P u_{0}$ for the equation $L \dot{u}=M u+f$. This solution has the form (2.1).

\section{§3. The Perturbed equation}

Let ker $L \neq\{0\}$, and let the operator $M$ be strongly $(L, p)$-radial [3, []. Applying the projection $Q$ to the two parts of (1.2) and using Remark 2.1, we obtain the equation

$$
L_{1} \dot{v}=M_{1} v+Q N(v+w),
$$

where $P u(t)=v(t),(I-P) u(t)=w(t), u(t)=v(t)+w(t)$. Therefore,

$$
\dot{v}=L_{1}^{-1} M_{1} v+L_{1}^{-1} Q N v+L_{1}^{-1} Q N w .
$$

Applying the operator $M_{0}^{-1}(I-Q)$ to equation (1.2), we get

$$
H \dot{w}=w+M_{0}^{-1} Q_{0} N w+M_{0}^{-1} Q_{0} N v
$$

where $Q_{0}=I-Q$. Thus, equation (1.2) reduces to the system (3.1), (3.2).

If the equation

$$
\dot{v}(t)=L_{1}^{-1} M_{1} v(t)+L_{1}^{-1} Q N_{1} v(t),
$$

where $N_{1}=\left.N\right|_{\mathfrak{U}^{1}}$, admits a resolving semigroup $\widetilde{U}^{t}, t \in \overline{\mathbb{R}}_{+}$, then the solution of the Cauchy problem

$$
v(0)=P u(0)=P u_{0}=v_{0}
$$

for equation (3.1) can be written in the form

$$
v(t)=\widetilde{U}^{t} v_{0}+\int_{0}^{t} \widetilde{U}^{t-s} L_{1}^{-1} Q N w(s) d s .
$$

Conditions sufficient for the existence of a $\left(C_{0}\right)$-continuous resolving semigroup for equation (3.3) were found by Phillips 9,14 in terms of perturbation theory for operator 
semigroups. Here the operator $L_{1}^{-1} Q N_{1}$ plays the role of the perturbing operator. To formulate these conditions, we need to introduce some additional notions.

Let $\mathfrak{V}$ be a Banach space, and let an operator $A \in \mathcal{C l}(\mathfrak{V})$ be the infinitesimal generator of a $\left(C_{0}\right)$-continuous semigroup of operators $\left\{V^{t} \in \mathcal{L}(\mathfrak{V}): t \in \overline{\mathbb{R}}_{+}\right\}$.

Definition 3.1. Let $\mathfrak{P}(A)$ denote the set of operators $B \in \mathcal{C l}(\mathfrak{V})$ satisfying the conditions

(i) $\operatorname{dom} B \supseteq \operatorname{dom} A$;

(ii) for any $t \in \mathbb{R}_{+}$there exists $C_{t} \in \mathbb{R}_{+}$such that for all $v$ in a subspace $\mathfrak{V}$ dense in $\mathfrak{V}$ we have

$$
\left\|B V^{t} v\right\|_{\mathfrak{V}} \leq C_{t}\|v\|_{\mathfrak{V}}
$$

(iii) in (ii), the constants $C_{t}$ can be chosen so that $\int_{0}^{1} C_{t} d t<\infty$.

By the results of [9, 14, if the Hille-Yosida conditions are fulfilled for the operator $L_{1}^{-1} M_{1}$, and if the operator $L_{1}^{-1} Q N_{1}$ belongs to the class $\mathfrak{P}\left(L_{1}^{-1} M_{1}\right)$, then equation (3.3) admits a $\left(C_{0}\right)$-continuous resolving semigroup. It is easy to check that this means precisely that the operator $N$ satisfies the following conditions:

(i) $\operatorname{dom} N \cap \mathfrak{U}^{1} \supseteq \operatorname{dom} M \cap \mathfrak{U}^{1}$;

(ii) for any $t \in \mathbb{R}_{+}$there exists $C_{t} \in \mathbb{R}_{+}$such that for all $u$ in a subspace $\mathfrak{U}^{1}$ dense in $\mathfrak{U}^{1}$ we have

$$
\left\|N U^{t} u\right\|_{\mathfrak{U}} \leq C_{t}\|u\|_{\mathfrak{U}}
$$

(iii) the constants $C_{t}$ in (ii) can be chosen so that $\int_{0}^{1} C_{t} d t<\infty$.

The set of all operators satisfying these three conditions will be denoted by $\mathfrak{P}(L, M)$.

Remark 3.1. If $N \in \mathcal{L}(\mathfrak{U} ; \mathfrak{F})$, then, obviously, $N \in \mathfrak{P}(L, M)$. By Theorem 2.1 (v), we can take $C_{t}=K e^{a t}\|N\|_{\mathcal{L}(\mathfrak{U} ; \mathfrak{F})}$.

Remark 3.2. If $N_{i} \in \mathfrak{P}(L, M), i=1,2$, then $N_{1}+N_{2} \in \mathfrak{P}(L, M)$.

We formulate the Phillips theorem on perturbation of a $\left(C_{0}\right)$-continuous semigroup in terms of equation (3.3).

Theorem 3.1 (14). Let $M$ be strongly $(L, p)$-radial, and let $N \in \mathfrak{P}(L, M)$. Then equation (3.3) admits a $\left(C_{0}\right)$-continuous semigroup of the form

$$
\left\{\widetilde{U}^{t}=\sum_{n=0}^{\infty} S_{n}(t) \in \mathcal{L}(\mathfrak{U}): t \in \overline{\mathbb{R}}_{+}\right\},
$$

where

$$
S_{0}(t)=U_{1}^{t}, \quad S_{n}(t) u=\int_{0}^{t} U_{1}^{t-s} L_{1}^{-1} Q N S_{n-1}(s) u d s, \quad u \in \mathfrak{U}^{1}, \quad t \in \overline{\mathbb{R}}_{+}, \quad n \in \mathbb{N} .
$$

Moreover, the series (3.6) converges absolutely and uniformly in $t$ on any finite interval in $\overline{\mathbb{R}}_{+}$, and the operator-valued functions $S_{n}(\cdot), n \in \mathbb{N}$, are strongly continuous in $t$ on $\overline{\mathbb{R}}_{+}$.

Application of this theorem allows us to establish the solvability of equation (3.1). The case of equation (3.2) is more difficult, because of the term $M_{0}^{-1} Q_{0} N w$. In [15], the case where $H=0,\left\|M_{0}^{-1} Q_{0} N_{0}\right\|<1$ was considered. However, in applications, as a rule, it is very hard to estimate the norm of such an operator. We shall consider the cases where this term vanishes. It is easily seen that this happens if $\mathfrak{U}^{0} \subset \operatorname{ker} N$ or $N\left[\mathfrak{U}^{0}\right] \subset \mathfrak{F}^{1}$. In the second case it is possible to express $w$ as a function of $v$ and of its derivatives of order at most $p$, because the operator $H$ is nilpotent. If we substitute this function in (3.5), we obtain a complicated high order integro-differential equation for $v$. We shall simplify the situation by assuming that $\operatorname{im} N \subset \mathfrak{F}^{1}$. Then it is easy to obtain the following result. 
Theorem 3.2. Suppose the operator $M$ is strongly $(L, p)$-radial, $N \in \mathfrak{P}(L, M)$, im $N \subset$ $\mathfrak{F}^{1}$, and $u_{0} \in \operatorname{dom} M_{1}$. Then there exists a unique solution $u \in C^{1}\left(\overline{\mathbb{R}}_{+} ; \mathfrak{U}\right)$ of problem (1.1), (1.2). This solution has the form $u(t)=\widetilde{U}^{t} P u_{0}$.

Proof. If im $N \subset \mathfrak{F}^{1}$, then $Q_{0} N=0$. In this case, equation (3.2) becomes $H \dot{w}(t)=w(t)$, so that it has a unique solution $w \equiv 0$ because $H$ is nilpotent (see [3, 6]). Therefore, the Cauchy problem $w(0)=(I-P) u_{0}$ for (3.2) has a solution only if $u_{0} \in \mathfrak{U}^{1}$, and then $u(t)=v(t)=\widetilde{U}^{t} P u_{0}$ is a solution of problem (1.1), (1.2).

Now we consider the first of the cases mentioned above: $\mathcal{U}^{0} \subset \operatorname{ker} N$.

Theorem 3.3. Suppose $M$ is strongly $(L, p)$-radial, $N \in \mathfrak{P}(L, M), \operatorname{dom} M \subset \operatorname{dom} N$, $\mathfrak{U}^{0} \subset \operatorname{ker} N, P u_{0} \in \operatorname{im} R_{(\mu, p)}^{L}(M)$, and

$$
(I-P) u_{0}=-\sum_{k=0}^{p} H^{k} M_{0}^{-1} Q_{0} N\left(L_{1}^{-1} M_{1}+L_{1}^{-1} Q N_{1}\right)^{k} P u_{0} .
$$

Then problem $(1.1),(1.2)$ admits a unique solution $u \in C^{1}\left(\overline{\mathbb{R}}_{+} ; \mathfrak{U}\right)$. This solution has the form

$$
u(t)=\widetilde{U}^{t} P u_{0}-\sum_{k=0}^{p} H^{k} M_{0}^{-1} Q_{0} N\left(L_{1}^{-1} M_{1}+L_{1}^{-1} Q N_{1}\right)^{k} \widetilde{U}^{t} P u_{0} .
$$

Proof. If $\mathfrak{U}^{0} \subset \operatorname{ker} N$, then we have $N w \equiv 0$. System (3.1), (3.2) takes the form

$$
\begin{aligned}
\dot{v}(t) & =L_{1}^{-1} M_{1} v(t)+L_{1}^{-1} Q N_{1} v(t), \\
H \dot{w}(t) & =w(t)+M_{0}^{-1} Q_{0} N v(t) .
\end{aligned}
$$

Then $v(t)=\widetilde{U}^{t} P u_{0}$ is a solution of problem (3.4), (3.8), and the function

$$
w(t)=-\sum_{k=0}^{p} H^{k} M_{0}^{-1} Q_{0} N v^{(k)}(t)=-\sum_{k=0}^{p} H^{k} M_{0}^{-1} Q_{0} N\left(L_{1}^{-1} M_{1}+L_{1}^{-1} Q N_{1}\right)^{k} \widetilde{U}^{t} P u_{0}
$$

solves equation (3.9) and the Cauchy problem

$$
w(0)=(I-P) u(0)=(I-P) u_{0}
$$

for (3.9), provided

$$
\begin{aligned}
(I-P) u_{0} & =-\sum_{k=0}^{p} H^{k} M_{0}^{-1} Q_{0} N\left(L_{1}^{-1} M_{1}+L_{1}^{-1} Q N_{1}\right)^{k} P u_{0}, \\
P u_{0} & \in \operatorname{dom}\left(L_{1}^{-1} M_{1}+L_{1}^{-1} Q N_{1}\right)^{p+1} .
\end{aligned}
$$

Since $N \in \mathfrak{P}(L, M)$, we have

$$
\operatorname{dom} N\left(L_{1}^{-1} M_{1}+L_{1}^{-1} Q N_{1}\right)^{p} \subset \operatorname{dom}\left(L_{1}^{-1} M_{1}+L_{1}^{-1} Q N_{1}\right)^{p+1} .
$$

Moreover, dom $L_{1}^{-1} M_{1} \subset \operatorname{dom} L_{1}^{-1} Q N_{1}$; consequently, $\operatorname{dom}\left(L_{1}^{-1} M_{1}+L_{1}^{-1} Q N_{1}\right)^{p+1}=$ $\operatorname{dom}\left(L_{1}^{-1} M_{1}\right)^{p+1}$. A vector $u$ belongs to $\operatorname{dom} L_{1}^{-1} M_{1}$ if and only if $u \in \operatorname{dom} M_{1}$ and there exists a vector $v \in \mathfrak{U}^{1}$ such that $M_{1} u=L_{1} v$. If $\lambda>a$, then $\left(M_{1}-\lambda L_{1}\right) u=$ $L_{1}(v-\lambda u)$ and $u=R_{\lambda}^{L_{1}}\left(M_{1}\right)(\lambda u-v) \in \operatorname{im} R_{\lambda}^{L_{1}}\left(M_{1}\right)$. Since this argument can be reversed, we have $\operatorname{dom} L_{1}^{-1} M_{1}=\operatorname{im} R_{\lambda}^{L_{1}}\left(M_{1}\right)$. Similarly, $\operatorname{dom}\left(L_{1}^{-1} M_{1}\right)^{p+1}=\operatorname{im} R_{(\mu, p)}^{L_{1}}\left(M_{1}\right)=$ $\operatorname{im} R_{(\mu, p)}^{L}(M)$. The second identity is fulfilled because $\operatorname{im} R_{(\mu, p)}^{L}(M) \subset \mathfrak{U}^{1}$ by the definition of the subspace $\mathfrak{U}^{1}$. Thus, condition (3.10) means precisely that the vector $P u_{0}$ belongs to the subspace $\operatorname{im} R_{(\mu, p)}^{L}(M)$. 
We have $P u_{0} \in \operatorname{dom} M$, and by Remark 2.2 the function $\widetilde{U}^{t} u_{0}$ is differentiable. The condition $\operatorname{dom} M \subset \operatorname{dom} N$ is necessary for the operator $N$ to be defined on the values of the solution.

The initial condition

$$
P u(0)=P u_{0}
$$

will be called the generalized Showalter condition. When reducing problem (1.2), (3.11) to an initial problem for system (3.1), (3.2), we arrive at the initial condition $v(0)=P u_{0}$ and have no constraints for $w(0)$. Therefore, the arguments used in the proofs of the last two theorems allow us to obtain similar solvability theorems for the generalized Showalter problem.

Theorem 3.4. Suppose $M$ is strongly $(L, p)$-radial, $N \in \mathfrak{P}(L, M), \operatorname{im} N \subset \mathfrak{F}^{1}$, and $u_{0} \in \mathfrak{U}^{0} \dot{+} \operatorname{dom} M_{1}$. Then there exists a unique solution $u \in C^{1}\left(\overline{\mathbb{R}}_{+} ; \mathfrak{U}\right)$ of problem $(1.2)$, (3.11). This solution has the form $u(t)=\widetilde{U}^{t} P u_{0}$.

Theorem 3.5. Suppose $M$ is strongly $(L, p)$-radial, $N \in \mathfrak{P}(L, M)$, $\operatorname{dom} M \subset \operatorname{dom} N$, $\mathfrak{U}^{0} \subset \operatorname{ker} N$, and $P u_{0} \in \operatorname{im} R_{(\mu, p)}^{L}(M)$. Then there exists a unique solution $u \in C^{1}\left(\overline{\mathbb{R}}_{+} ; \mathfrak{U}\right)$ of problem (1.2), (3.11). This solution has the form

$$
u(t)=\widetilde{U}^{t} P u_{0}-\sum_{k=0}^{p} H^{k} M_{0}^{-1} Q_{0} N\left(L_{1}^{-1} M_{1}+L_{1}^{-1} Q N_{1}\right)^{k} \widetilde{U}^{t} P u_{0} .
$$

Proof. In the proof, it is essential that equation (3.9) without any initial conditions is uniquely solvable. If we impose such conditions on a solution of this equation, then we need to coordinate the solution already available with the initial data. This leads to imposing additional constraints of the form (3.7) on the data of the problem. In the case of the generalized Showalter problem, we have no need to impose the constraint $(3.7)$.

\section{$\S 4$. The Inhomogeneous Perturbed EQUATION}

With the operators $L, M, N$ as above, we now consider the Cauchy problem and generalized Showalter problem for the inhomogeneous perturbed equation

$$
L \dot{u}(t)=M u(t)+N u(t)+f(t), \quad t \in \overline{\mathbb{R}}_{+},
$$

where $f: \overline{\mathbb{R}}_{+} \rightarrow \mathfrak{F}$.

Theorem 4.1. Suppose $M$ is strongly $(L, p)$-radial, $N \in \mathfrak{P}(L, M)$, $\operatorname{dom} M \subset \operatorname{dom} N$, $\operatorname{im} N \subset \mathfrak{F}^{1}, Q_{0} f \in C^{p+1}\left(\overline{\mathbb{R}}_{+} ; \mathfrak{F}\right), Q f-N \sum_{k=0}^{p} H^{k} M_{0}^{-1}\left(Q_{0} f\right)^{(k)} \in C^{1}\left(\overline{\mathbb{R}}_{+} ; \mathfrak{F}\right)$, and

$$
(I-P) u_{0}=-\sum_{k=0}^{p} H^{k} M_{0}^{-1}\left(Q_{0} f\right)^{k}(0) .
$$

Then the Cauchy problem $u(0)=u_{0}$ for equation (4.1) admits a unique solution $u \in$ $C^{1}\left(\overline{\mathbb{R}}_{+} ; \mathfrak{U}\right)$, and

$$
\begin{aligned}
u(t)=\widetilde{U}^{t} P u_{0} & +\int_{0}^{t} \widetilde{U}^{t-s} L_{1}^{-1} Q\left(f(s)-N \sum_{k=0}^{p} H^{k} M_{0}^{-1}\left(Q_{0} f\right)^{(k)}(s)\right) d s \\
& -\sum_{k=0}^{p} H^{k} M_{0}^{-1}\left(Q_{0} f\right)^{(k)}(t) .
\end{aligned}
$$


Proof. After application of the operator $M_{0}^{-1} Q_{0}$, equation (4.1) takes the form $H \dot{w}(t)=$ $w(t)+M_{0}^{-1} Q_{0} f(t)$. Since $H$ is nilpotent, the results of [3, 6] show that the function $-\sum_{k=0}^{p} H^{k} M_{0}^{-1}\left(Q_{0} f\right)^{(k)}(t)$ is a unique solution of the latter equation. Therefore, the Cauchy problem $w(0)=(I-P) u_{0}$ for it has a solution only if condition (4.2) is fulfilled.

Applying the operator $L_{1}^{-1} Q$ to (4.1), we deduce the equation

$$
\dot{v}(t)=L_{1}^{-1} M_{1} v(t)+L_{1}^{-1} Q N v(t)+L_{1}^{-1} Q N w(t)+L_{1}^{-1} Q f(t) .
$$

As a unique solution of the Cauchy problem $v(0)=P u_{0}$ for this equation we have the function

$$
\begin{aligned}
v(t) & =\widetilde{U}^{t} P u_{0}+\int_{0}^{t} \widetilde{U}^{t-s} L_{1}^{-1} Q(f(s)+N w(s)) d s \\
& =\widetilde{U}^{t} P u_{0}+\int_{0}^{t} \widetilde{U}^{t-s} L_{1}^{-1} Q\left(f(s)-N \sum_{k=0}^{p} H^{k} M_{0}^{-1}\left(Q_{0} f\right)^{(k)}(s)\right) d s .
\end{aligned}
$$

The solution $u(t)$ of the original problem is the sum of the two projections $v(t)$ and $w(t)$.

Theorem 4.2. Suppose $M$ is strongly $(L, p)$-radial, $N \in \mathfrak{P}(L, M)$, $\operatorname{dom} M \subset \operatorname{dom} N$, $\mathfrak{U}^{0} \subset \operatorname{ker} N, Q f \in C^{1}\left(\overline{\mathbb{R}}_{+} ; \mathfrak{F}\right), Q_{0}(N v+f) \in C^{p+1}\left(\overline{\mathbb{R}}_{+} ; \mathfrak{F}\right)$, where $v(t)=\widetilde{U}^{t} P u_{0}+$ $N \int_{0}^{t} \widetilde{U}^{t-s} L_{1}^{-1} Q f(s) d s$, and

$$
(I-P) u_{0}=-\sum_{k=0}^{p} H^{k} M_{0}^{-1}\left(Q_{0} N v+Q_{0} f\right)^{(k)}(0) .
$$

Then the Cauchy problem $u(0)=u_{0}$ for equation (4.1) admits a unique solution $u \in$ $C^{1}\left(\overline{\mathbb{R}}_{+} ; \mathfrak{U}\right)$, and

$$
u(t)=\widetilde{U}^{t} P u_{0}+\int_{0}^{t} \widetilde{U}^{t-s} L_{1}^{-1} Q f(s) d s-\sum_{k=0}^{p} H^{k} M_{0}^{-1}\left(Q_{0} N v+Q_{0} f\right)^{(k)}(t) .
$$

Proof. Since under the conditions of the theorem we have $N w \equiv 0$, equation (4.1) reduces to the system

$$
\begin{aligned}
\dot{v}(t) & =L_{1}^{-1} M_{1} v(t)+L_{1}^{-1} Q N_{1} v(t)+L_{1}^{-1} Q f(t), \\
H \dot{w}(t) & =w(t)+M_{0}^{-1} Q_{0} N v(t)+M_{0}^{-1} Q_{0} f(t) .
\end{aligned}
$$

The function

$$
v(t)=\widetilde{U}^{t} P u_{0}+\int_{0}^{t} \widetilde{U}^{t-s} L_{1}^{-1} Q f(s) d s
$$

is a unique solution of the Cauchy problem $v(0)=P u_{0}$ for the first equation in that system. Hence,

$$
w(t)=-\sum_{k=0}^{p} H^{k} M_{0}^{-1}\left(Q_{0} N v+Q_{0} f\right)^{(k)}(t)
$$

is a solution of the second equation. The Cauchy condition $w(0)=(I-P) u_{0}$ for the solution of (4.4) is satisfied if the coordination condition (4.3) is fulfilled.

In the case of a continuous perturbation operator $N$, the above result can be refined. Let $\operatorname{dom}\left(L_{1}^{-1} M_{1}+L_{1}^{-1} Q N_{1}\right)^{k}, k \in \mathbb{N}$, be equipped with the graph norm of the operator $\left(L_{1}^{-1} M_{1}+L_{1}^{-1} Q N_{1}\right)^{k}$. It is well known that such a subspace is a Banach space if the corresponding operator is closed. By $\operatorname{dom}\left(L_{1}^{-1} M_{1}+L_{1}^{-1} Q N_{1}\right)^{0}$ we shall mean the Banach space $\mathfrak{U}$. 
Theorem 4.3. Suppose $M$ is strongly $(L, p)$-radial, $N \in \mathcal{L}(\mathfrak{U} ; \mathfrak{F}), \mathfrak{U}^{0} \subset \operatorname{ker} N, Q_{0} f \in$ $C^{p+1}\left(\overline{\mathbb{R}}_{+} ; \mathfrak{F}\right), Q f \in C^{1}\left(\overline{\mathbb{R}}_{+} ; \mathfrak{F}\right), P u_{0} \in \operatorname{im} R_{(\mu, p)}^{L}(M)$,

$$
L_{1}^{-1} Q f \in \bigcap_{k=0}^{p} C^{p-k}\left(\overline{\mathbb{R}}_{+} ; \operatorname{dom}\left(L_{1}^{-1} M_{1}+L_{1}^{-1} Q N_{1}\right)^{k}\right),
$$

and

$$
\begin{aligned}
(I-P) u_{0}= & -\sum_{k=0}^{p} H^{k} M_{0}^{-1} Q_{0} N\left(L_{1}^{-1} M_{1}+L_{1}^{-1} Q N_{1}\right)^{k} P u_{0}-\sum_{k=0}^{p} H^{k} M_{0}^{-1}\left(Q_{0} f\right)^{(k)}(0) \\
& -\sum_{k=1}^{p} H^{k} M_{0}^{-1} Q_{0} N \sum_{m=0}^{k-1}\left(L_{1}^{-1} M_{1}+L_{1}^{-1} Q N_{1}\right)^{m}\left(L_{1}^{-1} Q f\right)^{(k-1-m)}(0) .
\end{aligned}
$$

Then the Cauchy problem $u(0)=u_{0}$ for equation (4.1) admits a unique solution $u \in$ $C^{1}(\overline{\mathbb{R}} ; \mathfrak{U})$, and

$$
\begin{aligned}
u(t)=\widetilde{U}^{t} P u_{0} & +\int_{0}^{t} \widetilde{U}^{t-s} L_{1}^{-1} Q f(s) d s-\sum_{k=0}^{p} H^{k} M_{0}^{-1}\left(Q_{0} f\right)^{(k)}(t) \\
& -\sum_{k=0}^{p} H^{k} M_{0}^{-1} Q_{0} N\left(L_{1}^{-1} M_{1}+L_{1}^{-1} Q N_{1}\right)^{k} \widetilde{U}^{t} P u_{0} \\
& -\sum_{k=0}^{p} H^{k} M_{0}^{-1} Q_{0} N \int_{0}^{t}\left(L_{1}^{-1} M_{1}+L_{1}^{-1} Q N_{1}\right)^{k} \widetilde{U}^{t-s} L_{1}^{-1} Q f(s) d s \\
& -\sum_{k=1}^{p} H^{k} M_{0}^{-1} Q_{0} N \sum_{m=0}^{k-1}\left(L_{1}^{-1} M_{1}+L_{1}^{-1} Q N_{1}\right)^{m}\left(L_{1}^{-1} Q f\right)^{(k-1-m)}(t) .
\end{aligned}
$$

Proof. If we write the function $v(t)$ obtained in the proof of the preceding theorem explicitly and recall that the operator $N$ is continuous, we arrive at formula (4.5). The identity

$$
\begin{aligned}
& \frac{d^{k}}{d t^{k}} \int_{0}^{t} \widetilde{U}^{t-s} L_{1}^{-1} Q f(s) d s \\
& \quad=\left(L_{1}^{-1} Q f\right)^{(k-1)}(t)+\left(L_{1}^{-1} M_{1}+L_{1}^{-1} Q N_{1}\right)\left(L_{1}^{-1} Q f\right)^{(k-2)}(t)+\cdots \\
& \quad+\left(L_{1}^{-1} M_{1}+L_{1}^{-1} Q N_{1}\right)^{k-1} L_{1}^{-1} Q f(t)+\int_{0}^{t}\left(L_{1}^{-1} M_{1}+L_{1}^{-1} Q N_{1}\right)^{k} \widetilde{U}^{t-s} L_{1}^{-1} Q f(s) d s
\end{aligned}
$$

should also be used.

As in $\S 3$, these theorems imply sufficient conditions for the solvability of the generalized Showalter problem. The difference from similar conditions for the Cauchy problem is that the coordination condition (4.2) or (4.3) is absent. The solution of the generalized Showalter problem has the same form as that for the Cauchy problem.

\section{§5. Equations With polynomials in Elliptic SElfadjoint operators}

Let $P_{n}(\lambda)=\sum_{i=0}^{n} c_{i} \lambda^{i}$ and $Q_{m}(\lambda)=\sum_{j=0}^{m} d_{j} \lambda^{j}$ be polynomials such that $c_{i}, d_{j} \in \mathbb{C}$, $i=0, \ldots, n, j=0, \ldots, m, c_{n}, d_{m} \neq 0, m \geq n$. Next, let $\Omega \subset \mathbb{R}^{s}$ be a bounded domain with boundary $\partial \Omega$ of class $C^{\infty}$, and let $A, B_{1}, \ldots, B_{r}$ be a regularly elliptic collection 
of operators [16, where

$$
\begin{aligned}
(A u)(x) & =\sum_{|\alpha| \leq 2 r} a_{\alpha}(x) D^{\alpha} u(x), \quad a_{\alpha} \in C^{\infty}(\bar{\Omega}), \\
\left(B_{l} u\right)(x) & =\sum_{|\alpha| \leq r_{l}} b_{l \alpha}(x) D^{\alpha} u(x), \quad b_{l \alpha} \in C^{\infty}(\partial \Omega), \quad l=1, \ldots, r .
\end{aligned}
$$

It is also assumed that the operator $A_{1} \in \mathcal{C l}\left(L_{2}(\Omega)\right)$ with domain $\operatorname{dom} A_{1}=H_{\left\{B_{l}\right\}}^{2 r}(\Omega)$ [16], acting as $A_{1} u=A u$, is selfadjoint.

Suppose $K \in L_{2}(\Omega \times \Omega)$; then, by the Fubini theorem, for almost all $x, y \in \Omega$ we have $K(\cdot, y), K(x, \cdot) \in L_{2}(\Omega)$. We want to reduce the initial boundary value problem

$$
\begin{aligned}
P_{n}(A) u_{t}(x, t) & =Q_{m}(A) u(x, t)+\int_{\Omega} K(x, y) u(y, t) d y, \quad(x, t) \in \Omega \times \overline{\mathbb{R}}_{+}, \\
B_{l} A^{k} u(x, t) & =0, \quad k=1, \ldots, r, \quad l=1, \ldots, r, \quad(x, t) \in \partial \Omega \times \overline{\mathbb{R}}_{+}, \\
u(x, 0) & =u_{0}(x), \quad x \in \Omega,
\end{aligned}
$$

to a problem of the form (1.1), (1.2). For this, we take

$$
\begin{aligned}
\mathfrak{U} & =\left\{u \in H^{2 r n}(\Omega): B_{l} A^{k} u(x)=0, \quad k=0, \ldots, n-1, l=1, \ldots, r, x \in \partial \Omega\right\}, \\
\mathfrak{F} & =L_{2}(\Omega), \quad L=P_{n}(A), \quad M=Q_{m}(A), \\
\operatorname{dom} M & =\left\{u \in H^{2 r m}(\Omega): B_{l} A^{k} u(x)=0, k=0, \ldots, m-1, l=1, \ldots, r, x \in \partial \Omega\right\}, \\
(N u)(x) & =\int_{\Omega} K(x, y) u(y) d y, \quad N \in \mathcal{L}(\mathfrak{U} ; \mathfrak{F}), \quad\|N\|_{\mathcal{L}(\mathfrak{U} ; \mathfrak{F})} \leq\|K\|_{L_{2}(\Omega \times \Omega) .}
\end{aligned}
$$

Indeed, by the Hölder inequality we have

$$
\begin{aligned}
\|N u\|_{L_{2}(\Omega)}^{2} & =\int_{\Omega}\left|\int_{\Omega} K(x, y) u(y) d y\right|^{2} d x \leq \int_{\Omega}\left(\int_{\Omega}|K(x, y)|^{2} d y \int_{\Omega}|u(y)|^{2} d y\right) d x \\
& =\int_{\Omega} \int_{\Omega}|K(x, y)|^{2} d x d y \cdot\|u\|_{L_{2}(\Omega)}^{2} .
\end{aligned}
$$

Let $\left\{\varphi_{k}: k \in \mathbb{N}\right\}$ denote the orthonormal eigenfunctions of the operator $A_{1}$ with respect to the inner product $\langle\cdot, \cdot\rangle$ in $L_{2}(\Omega)$, enumerated with respect to the nonascending order of the corresponding eigenvalues $\left\{\lambda_{k}: k \in \mathbb{N}\right\}$ (with regard to multiplicity). Here we use the fact that the spectrum of $A_{1}$ is real and tends to $-\infty$.

Theorem 5.1. Suppose $m>n,(-1)^{m-n} \operatorname{Re}\left(d_{m} / c_{n}\right) \leq 0$, and the spectrum $\sigma\left(A_{1}\right)$ contains no common roots of the polynomials $P_{n}(\lambda)$ and $Q_{m}(\lambda)$. Then the operator $M$ is strongly $(L, 0)$-radial.

Proof. Under the conditions of the theorem, all numbers $\mu_{k}=Q_{m}\left(\lambda_{k}\right) / P_{n}\left(\lambda_{k}\right)$ with $k$ such that $P_{n}\left(\lambda_{k}\right) \neq 0$ form the set $\sigma^{L}(M)$. Moreover,

$$
\lim _{k \rightarrow \infty}\left|\arg \frac{Q_{m}\left(\lambda_{k}\right)}{P_{n}\left(\lambda_{k}\right)}\right| \in[\pi / 2, \pi] .
$$

Therefore there exists $a \geq 0$ such that $\sigma^{L}(M) \subset\{\mu \in \mathbb{C}: \operatorname{Re} \mu \leq a\}$. We must check the estimates in the definition of the strong $(L, 0)$-radiality. Suppose $\mu, \nu>a, u \in \mathfrak{U}$, and 
$f \in L_{2}(\Omega)$; then

$$
\begin{aligned}
& \left\|L_{\mu}^{L}(M) f\right\|_{L_{2}(\Omega)}^{2}=\sum_{P_{n}\left(\lambda_{k}\right) \neq 0} \frac{\left|\left\langle f, \varphi_{k}\right\rangle\right|^{2}}{\left|\mu-\frac{Q_{m}\left(\lambda_{k}\right)}{P_{n}\left(\lambda_{k}\right)}\right|^{2}} \leq \frac{\|f\|_{L_{2}(\Omega)}^{2}}{(\mu-a)^{2}}, \\
& \left\|R_{\mu}^{L}(M) u\right\|_{H^{2 r n}(\Omega)}^{2}=\sum_{P_{n}\left(\lambda_{k}\right) \neq 0} \frac{\left(1+\lambda_{k}^{2 n}\right)\left|\left\langle u, \varphi_{k}\right\rangle\right|^{2}}{\left|\mu-\frac{Q_{m}\left(\lambda_{k}\right)}{P_{n}\left(\lambda_{k}\right)}\right|^{2}} \leq \frac{\|u\|_{H^{2 r n}(\Omega)}^{2}}{(\mu-a)^{2}}, \\
& \left\|R_{\mu}^{L}(M)(\nu L-M)^{-1} f\right\|_{H^{2 r n}(\Omega)}^{2} \\
& \quad=\sum_{P_{n}\left(\lambda_{k}\right) \neq 0} \frac{\left(1+\lambda_{k}^{2 n}\right)\left|\left\langle f, \varphi_{k}\right\rangle\right|^{2}}{\left|P_{n}\left(\lambda_{k}\right)\right|^{2}\left|\mu-\frac{Q_{m}\left(\lambda_{k}\right)}{P_{n}\left(\lambda_{k}\right)}\right|^{2}\left|\nu-\frac{Q_{m}\left(\lambda_{k}\right)}{P_{n}\left(\lambda_{k}\right)}\right|^{2}} \leq \frac{C\|f\|_{L_{2}(\Omega)}^{2}}{(\mu-a)^{2}(\nu-a)^{2}} .
\end{aligned}
$$

Indeed, since $\lim _{k \rightarrow \infty} \frac{1+\lambda_{k}^{2}}{\left.P_{n}\left(\lambda_{k}\right)\right|^{2}}=\left|c_{n}\right|^{-2}$, the sequence is bounded by some constant $C>0$. Taking $f \in \operatorname{dom} M=\grave{F}$, we obtain

$$
\begin{aligned}
& \left\|M(\nu L-M)^{-1} L_{\mu}^{L}(M) f\right\|_{L_{2}(\Omega)}^{2} \\
& \quad=\sum_{P_{n}\left(\lambda_{k}\right) \neq 0} \frac{\left|Q_{m}\left(\lambda_{k}\right)\right|^{2}\left|\left\langle f, \varphi_{k}\right\rangle\right|^{2}}{\left|P_{n}\left(\lambda_{k}\right)\right|^{2}\left|\mu-\frac{Q_{m}\left(\lambda_{k}\right)}{P_{n}\left(\lambda_{k}\right)}\right|^{2}\left|\nu-\frac{Q_{m}\left(\lambda_{k}\right)}{P_{n}\left(\lambda_{k}\right)}\right|^{2}} \leq \frac{c^{-2}\|M f\|_{L_{2}(\Omega)}^{2}}{(\mu-a)^{2}(\nu-a)^{2}} .
\end{aligned}
$$

We have used the inequality $\left|P_{n}\left(\lambda_{k}\right)\right| \geq c$ for all $k$ occurring in the sum, which follows from the absence of finite limit points of the set $\left\{\lambda_{k}\right\}$. Moreover, in the case under consideration we have $\operatorname{dom} M \subset \mathfrak{U} \subset \mathfrak{F}=L_{2}(\Omega)$.

Remark 5.1. In Theorem 5.1 and in the subsequent statements, the conditions $m>n$, $(-1)^{m-n} \operatorname{Re}\left(d_{m} / c_{n}\right) \leq 0$ can be replaced by the condition $m=n$.

We have $P=Q=\sum_{P_{n}\left(\lambda_{k}\right) \neq 0}\left\langle\cdot, \varphi_{k}\right\rangle \varphi_{k}$, the subspaces $\mathfrak{U}^{0}=\mathfrak{F}^{0}=\operatorname{span}\left\{\varphi_{k}: P_{n}\left(\lambda_{k}\right)=0\right\}$ are finite-dimensional, and $\mathfrak{U}^{1}, \mathfrak{F}^{1}$ are the closures of the set $\operatorname{span}\left\{\varphi_{k}: P_{n}\left(\lambda_{k}\right) \neq 0\right\}$ with respect to the norm of the space $\mathfrak{U}$ or $\mathfrak{F}$, respectively.

Theorem 5.2. Suppose $m>n,(-1)^{m-n} \operatorname{Re}\left(d_{m} / c_{n}\right) \leq 0$, the spectrum $\sigma\left(A_{1}\right)$ contains no common roots of the polynomials $P_{n}(\lambda)$ and $Q_{m}(\lambda), u_{0} \in \operatorname{dom} M$, and for all $k \in \mathbb{N}$ such that $P_{n}\left(\lambda_{k}\right)=0$ we have

$$
\int_{\Omega} K(x, y) \varphi_{k}(x) d x \equiv 0, \quad \int_{\Omega} u_{0}(x) \varphi_{k}(x) d x=0 .
$$

Then the problem (5.1)-(5.3) is uniquely solvable.

Proof. By Theorem 5.1 and Remark 3.1, the assumptions of Theorem 3.2 are fulfilled. Conditions (5.4) mean that $\operatorname{im} N \subset \mathfrak{F}^{1}, u_{0} \in \mathfrak{U}^{1}$. Indeed, since $K(x, y) \varphi_{k}(x) u(y) \in$ $L_{2}(\Omega \times \Omega)$, the Fubini theorem says that

$$
\int_{\Omega} \varphi_{k}(x) d x \int_{\Omega} K(x, y) u(y) d y=\int_{\Omega} u(y) d y \int_{\Omega} K(x, y) \varphi_{k}(x) d x
$$

The last integral is equal to zero for all $k \in \mathbb{N}$ such that $P_{n}\left(\lambda_{k}\right)=0$. This means that for all $u \in \mathfrak{U}$ the functions $N u$ are $L_{2}(\Omega)$-orthogonal to the functions $\varphi_{k}$ with the corresponding $k$. Therefore, $\operatorname{im} N \perp \mathfrak{F}^{0}$.

Remark 5.2. Clearly, for system (5.1), (5.2) the condition $u_{0} \in \operatorname{dom} M$ can be formulated in terms of the Fourier coefficients $u_{0 k}=\left\langle u_{0}, \varphi_{k}\right\rangle$ as follows: $\left\{\lambda_{k}^{m} u_{0 k}\right\} \in l_{2}$. 
Consider the case where $P_{1}(\lambda)=1+\lambda, Q_{2}(\lambda)=\lambda-2 \lambda^{2}, \Omega=(0, \pi), A u=u^{\prime \prime}, r=1$, and $B_{1}=I$. Then $\lambda_{k}=-k^{2}, \varphi_{k}(x)=\sin k x, k \in \mathbb{N}$ and problem (5.1)-(5.3) takes the form

$$
\begin{aligned}
\left(1+\frac{\partial^{2}}{\partial x^{2}}\right) \frac{\partial u}{\partial t}(x, t)= & \left(\frac{\partial^{2}}{\partial x^{2}}-2 \frac{\partial^{4}}{\partial x^{4}}\right) u(x, t) \\
& +\int_{0}^{\pi} K(x, y) u(y, t) d y, \quad(x, t) \in(0, \pi) \times \overline{\mathbb{R}}_{+}, \\
u(0, t)= & \frac{\partial^{2} u}{\partial x^{2}}(0, t)=u(\pi, t)=\frac{\partial^{2} u}{\partial x^{2}}(\pi, t)=0, \quad t \in \overline{\mathbb{R}}_{+}, \\
u(x, 0)= & u_{0}(x), \quad x \in(0, \pi) .
\end{aligned}
$$

For this problem, conditions (5.4) read as follows:

$$
\int_{0}^{\pi} K(x, y) \sin x d x=0, \quad \int_{0}^{\pi} u_{0}(x) \sin x d x=0,
$$

and they are fulfilled, e.g., if $K(x, y)=\sum_{k=2}^{q} a_{k}(y) \sin k x$, where $a_{k} \in L_{2}(0, \pi), u_{0}(x)=$ $\sum_{k=2}^{\infty} b_{k} \sin k x$. The condition $\left\{k^{4} b_{k}\right\} \in l_{2}$ guarantees that $u_{0} \in \operatorname{dom} M \subset H^{4}(0, \pi)$.

Lemma 5.1. Suppose $M$ is strongly $(L, 0)$-radial. Then the conditions $u_{0} \in \mathfrak{U}^{0} \dot{+} \operatorname{dom} M_{1}$ and $P u_{0} \in \operatorname{im} R_{(\mu, p)}^{L}(M)$ are equivalent. For system (5.1), (5.2), they are equivalent to the condition $u_{0} \in \operatorname{dom} M$.

Proof. Since $p=0$, we have

$$
\operatorname{im} R_{\mu}^{L}(M)=\operatorname{im} R_{\mu}^{L_{1}}\left(M_{1}\right)=\operatorname{dom} L_{1}^{-1} M_{1}=\operatorname{dom} M_{1} .
$$

For system (5.1), (5.2), since the subspace $\mathfrak{U}^{0}$ is finite-dimensional, we have dom $M_{0}=\mathfrak{U}^{0}$. Therefore, $\operatorname{dom} M=\operatorname{dom} M_{0} \dot{+} \operatorname{dom} M_{1}=\mathfrak{U}^{0} \dot{+} \operatorname{dom} M_{1}$.

If the operator $M$ is strongly $(L, 0)$-radial, then $\mathfrak{U}^{0}=\operatorname{ker} L$ [3, 6]. Consequently, the generalized Showalter problem is equivalent to the Showalter problem $L u(0)=L u_{0}[17$. Therefore, the generalized Showalter condition for this system can be written in the form

$$
P_{n}(A) u(x, 0)=P_{n}(A) u_{0}(x), \quad x \in \Omega .
$$

Denote $u_{0 k}=\left\langle u_{0}, \varphi_{k}\right\rangle, k \in \mathbb{N}$. Applying Theorems 3.4 and 3.5, we obtain the following assertions.

Theorem 5.3. Suppose $m>n,(-1)^{m-n} \operatorname{Re}\left(d_{m} / c_{n}\right) \leq 0$, the spectrum $\sigma\left(A_{1}\right)$ contains no common roots of the polynomials $P_{n}(\lambda)$ and $Q_{m}(\lambda), u_{0} \in \operatorname{dom} M$, and for all $k \in \mathbb{N}$ such that $P_{n}\left(\lambda_{k}\right)=0$ we have $\int_{\Omega} K(x, y) \varphi_{k}(x) d x \equiv 0$. Then problem (5.1), (5.2), (5.8) has a unique solution $u \in C^{1}\left(\overline{\mathbb{R}}_{+} ; \mathfrak{U}\right)$.

Theorem 5.4. Suppose $m>n,(-1)^{m-n} \operatorname{Re}\left(d_{m} / c_{n}\right) \leq 0$, the spectrum $\sigma\left(A_{1}\right)$ contains no common roots of the polynomials $P_{n}(\lambda)$ and $Q_{m}(\lambda), u_{0} \in \operatorname{dom} M$, and for all $k \in \mathbb{N}$ such that $P_{n}\left(\lambda_{k}\right)=0$ we have $\int_{\Omega} K(x, y) \varphi_{k}(y) d y \equiv 0$. Then there exists a unique solution $u \in C^{1}\left(\overline{\mathbb{R}}_{+} ; \mathfrak{U}\right)$ of problem $(5.1),(5.2),(5.8)$.

Proof. The integral identity in the conditions of the theorem means that $\mathfrak{U}^{0} \subset \operatorname{ker} N$. By Lemma 5.1, $P u_{0} \in \operatorname{im} R_{\mu}^{L}(M)$ if and only if $u \in \operatorname{dom} M$. Applying Theorem 3.5, we get the claim.

Now, consider the same problem for $m>2 n$, but with a differential perturbation operator

$$
P_{n}(A) u_{t}(x, t)=Q_{m}(A) u(x, t)+P_{n}(A) \sum_{|\alpha| \leq(m-2 n) 2 r-1} a_{\alpha}(x) D^{\alpha} u(x, t), \quad(x, t) \in \Omega \times \overline{\mathbb{R}}_{+} .
$$


Here we use the standard notation $\alpha=\left(\alpha_{1}, \ldots, \alpha_{s}\right) \in \mathbb{N}_{0}^{s},|\alpha|=\alpha_{1}+\cdots+\alpha_{s}, D^{\alpha}=$ $\frac{\partial^{|\alpha|}}{\partial x_{1}^{\alpha_{1}} \cdots \partial x_{s}^{\alpha_{s}}}$. The functions $a_{\alpha}$ belong to $L_{\infty}(\Omega)$.

Lemma 5.2. Under the conditions of the present section,

$$
N=P_{n}(A) \sum_{|\alpha| \leq(m-2 n) 2 r-1} a_{\alpha}(x) D^{\alpha} \in \mathfrak{P}(L, M) .
$$

Proof. We have $\operatorname{dom} M \subset \operatorname{dom} N=H^{(m-n) 2 r-1}(\Omega)$, and for $u \in \mathfrak{U}$ we can write

$$
\begin{aligned}
N U^{t} u & =P_{n}(A) \sum_{|\alpha| \leq(m-2 n) 2 r-1} a_{\alpha}(x) D^{\alpha} \sum_{k=1}^{\infty} e^{\mu_{k} t}\left\langle u, \varphi_{k}\right\rangle \varphi_{k} \\
& =N\left(b+1-A_{1}\right)^{n-m+1 / r} \sum_{k=1}^{\infty} \frac{\left(b+1-\lambda_{k}\right)^{m-n-1 / r}}{\mu_{k}^{1-\frac{1}{(m-n) r}}} \mu_{k}^{1-\frac{1}{(m-n) r}} e^{\mu_{k} t}\left\langle u, \varphi_{k}\right\rangle \varphi_{k},
\end{aligned}
$$

where $\mu_{k}=\frac{Q_{m}\left(\lambda_{k}\right)}{P_{n}\left(\lambda_{k}\right)}$ and $b=\max _{k \in \mathbb{N}} \lambda_{k}$. Since the operator $A_{1}$ is sectorial, the set $\operatorname{dom}\left(b+1-A_{1}\right)^{\beta}$ is a Banach space with the norm

$$
\|u\|_{\beta}=\left\|\left(b+1-A_{1}\right)^{\beta} u\right\|_{L_{2}(\Omega)} .
$$

If $\beta>m-n-\frac{1}{2 r}$, then this space is continuously and densely embedded in $H^{(m-n) 2 r-1}(\Omega)$ (Theorems 1.4.8 and 1.6.1 in [18]); moreover,

$$
(b+1-A)^{n-m+1 / r}: L_{2}(\Omega) \rightarrow \operatorname{dom}\left(b+1-A_{1}\right)^{m-n-1 / r} .
$$

Therefore, for $u \in L_{2}(\Omega)$ we have

$$
\begin{aligned}
& \left\|N\left(b+1-A_{1}\right)^{n-m+1 / r} u\right\|_{L_{2}(\Omega)} \\
& \quad \leq \sup _{|\alpha| \leq(m-2 n) 2 r-1}\left\|a_{\alpha}\right\|_{L_{\infty}(\Omega)}\left\|\left(b+1-A_{1}\right)^{n-m+1 / r} u\right\|_{H^{(m-n) 2 r-1}(\Omega)} \\
& \quad \leq c\left\|\left(b+1-A_{1}\right)^{n-m+1 / r} u\right\|_{m-n-1 / r}=c\|u\|_{L_{2}(\Omega)} .
\end{aligned}
$$

Since the limit

$$
\lim _{k \rightarrow \infty} \frac{\left(b+1-\lambda_{k}\right)^{m-n-1 / r}}{\mu_{k}^{1-\frac{1}{(m-n) r}}}=\lim _{k \rightarrow \infty} \frac{\left(b+1-\lambda_{k}\right)^{m-n-1 / r}}{\left(\frac{d_{m}}{c_{n}} \lambda_{k}^{m-n}\right)^{1-\frac{1}{(m-n) r}}}
$$

is finite, we have

$$
\begin{aligned}
& \left\|\sum_{k=1}^{\infty} \frac{\left(b+1-\lambda_{k}\right)^{m-n-1 / r}}{\mu_{k}^{1-\frac{1}{(m-n) r}}} \mu_{k}^{1-\frac{1}{(m-n) r}} e^{\mu_{k} t}\left\langle u, \varphi_{k}\right\rangle \varphi_{k}\right\|_{L_{2}(\Omega)} \\
& \leq c\left\|\sum_{k=1}^{\infty} \mu_{k}^{1-\frac{1}{(m-n) r}} e^{\mu_{k} t}\left\langle u, \varphi_{k}\right\rangle \varphi_{k}\right\|_{L_{2}(\Omega)} \leq c t^{\frac{1}{(m-n) r}-1}\|u\|_{L_{2}(\Omega)} .
\end{aligned}
$$

We have used the fact that for positive $t$ the function $\mu^{\beta} e^{\mu t}$ of the variable $\mu$ has a unique local minimum, equal to $c t^{-\beta}$, at the point $\mu=-\beta / t$. Therefore, on the semiaxis $(-\infty, a]$ the maximum of the modulus of this function is attained at that point or at the point $\mu=a$.

Finally, we obtain $\left\|N U^{t}\right\|_{\mathcal{L}\left(L_{2}(\Omega)\right)} \leq c t^{\frac{1}{(m-n) r}-1}$.

Theorem 5.5. Suppose $m>2 n,(-1)^{m-n} \operatorname{Re}\left(d_{m} / c_{n}\right) \leq 0$, the spectrum $\sigma\left(A_{1}\right)$ contains no common roots of the polynomials $P_{n}(\lambda)$ and $Q_{m}(\lambda), u_{0} \in \operatorname{dom} M$, and for all $k \in \mathbb{N}$ such that $P_{n}\left(\lambda_{k}\right)=0$ we have $\int_{\Omega} u_{0}(x) \varphi_{k}(x) d x=0$. Then problem (5.2), (5.3), (5.9) has a unique solution. 
Proof. The proof is similar to that of Theorem 5.2. Note that the conditions imposed on the operator $N$ in Theorem 3.2 are fulfilled by Lemma 5.2 , because, obviously, $\mathfrak{F}^{1}=\operatorname{im} L$ implies $\operatorname{im} N \subset \mathfrak{F}^{1}$.

Remark 5.3. For equation (5.9), the conditions $\operatorname{im} N \subset \mathfrak{F}^{1}$ and $\mathfrak{U}^{0} \subset \operatorname{ker} N$ are fulfilled simultaneously. Therefore, by the obvious embedding $\operatorname{dom} M \subset \operatorname{dom} N$ and Lemma 5.1, an application of Theorem 3.4 or Theorem 3.5 to problem (5.2), (5.8), (5.9) leads to the following result.

Theorem 5.6. Suppose $m>2 n,(-1)^{m-n} \operatorname{Re}\left(d_{m} / c_{n}\right) \leq 0$, the spectrum $\sigma\left(A_{1}\right)$ contains no common roots of the polynomials $P_{n}(\lambda)$ and $Q_{m}(\lambda)$, and $u_{0} \in \operatorname{dom} M$. Then problem (5.2), (5.8), (5.9) has a unique solution $u \in C^{1}\left(\overline{\mathbb{R}}_{+} ; \mathfrak{U}\right)$.

Remark 5.4. It is clear that, using Remark 3.2 , it is possible to obtain solvability conditions of similar initial boundary problems for the equation

$$
P_{n}(A) u_{t}(x, t)=Q_{m}(A) u(x, t)+P_{n}(A) \sum_{|\alpha| \leq(m-2 n) 2 r-1} a_{\alpha}(x) D^{\alpha} u(x, t)+\int_{\Omega} K(x, y) u(y, t) d y
$$

containing differential and integral perturbations simultaneously.

Remark 5.5. The Barenblatt-Zheltov-Kochina equation, the Dzektser equation, and other equations occurring in applications (see [1, 2, 3, 4, 6, ) are particular cases of the nonperturbed equation of the form (5.1).

\section{§6. Linearized SyStem OF THE PHASE FIELD EQUATIONS}

Let $\Omega \subset \mathbb{R}^{s}$ be a bounded domain with boundary $\partial \Omega$ of class $C^{\infty}$, and let $\lambda, \alpha, \beta \in \mathbb{R}$, $K_{m} \in L_{2}(\Omega \times \Omega), m=1,2, \quad a_{i}, b_{j} \in L_{\infty}(\Omega), i, j=0, \ldots, s$. Consider the problem

$$
\begin{aligned}
& u(x, 0)= u_{0}(x), \quad x \in \Omega \\
& \frac{\partial u}{\partial n}(x, t)+ \lambda u(x, t)=\frac{\partial v}{\partial n}(x, t)+\lambda v(x, t)=0, \quad(x, t) \in \partial \Omega \times \overline{\mathbb{R}}_{+}, \\
& u_{t}(x, t)= \Delta u(x, t)-\Delta v(x, t)+\int_{\Omega} K_{1}(x, y) u(y, t) d y+\int_{\Omega} K_{2}(x, y) v(y, t) d y \\
&+a_{0}(x) u+\sum_{i=1}^{s} a_{i}(x) \frac{\partial u}{\partial x_{i}}+b_{0}(x) v+\sum_{j=1}^{s} b_{j}(x) \frac{\partial v}{\partial x_{j}}, \quad(x, t) \in \Omega \times \overline{\mathbb{R}}_{+}, \\
& \Delta v(x, t)+\beta v(x, t)+\alpha u(x, t)=0, \quad(x, t) \in \Omega \times \overline{\mathbb{R}}_{+} .
\end{aligned}
$$

Here the role of the unknowns is played by the functions $u(x, t), v(x, t)$.

Remark 6.1. System (6.1)-(6.4) with $K_{m} \equiv 0, m=1,2$, is a linearization at zero, up to linear changes, of the initial boundary value problem for the system of the phase field equations describing the first kind phase transitions in mesoscopic theory [19, 20].

We put $\mathfrak{U}=\mathfrak{F}=\left(L_{2}(\Omega)\right)^{2}$,

$$
\begin{gathered}
L=\left(\begin{array}{cc}
1 & 0 \\
0 & 0
\end{array}\right), \quad M=\left(\begin{array}{cc}
\Delta & -\Delta \\
\alpha & \beta+\Delta
\end{array}\right), \\
H_{\frac{\partial}{\partial n}+\lambda}^{2}(\Omega)=\left\{w \in H^{2}(\Omega):\left(\frac{\partial}{\partial n}+\lambda\right) w(x)=0, x \in \partial \Omega\right\}, \operatorname{dom} M=\left(H_{\frac{\partial}{\partial n}+\lambda}^{2}(\Omega)\right)^{2}, \\
N_{1}\left(\begin{array}{l}
u \\
v
\end{array}\right)=\left(\begin{array}{cc}
\int_{\Omega} K_{1}(x, y) u(y) d y+\int_{\Omega} K_{2}(x, y) v(y) d y \\
0
\end{array}\right), \quad N_{1} \in \mathcal{L}(\mathfrak{U}), \\
N_{2}=\left(\begin{array}{cc}
a_{0}(x)+\sum_{i=1}^{s} a_{i}(x) \frac{\partial}{\partial x_{i}} & b_{0}(x)+\sum_{j=1}^{s} b_{j}(x) \frac{\partial}{\partial x_{j}} \\
0 & 0
\end{array}\right),
\end{gathered}
$$


$N=N_{1}+N_{2}, \operatorname{dom} N=\operatorname{dom} N_{2}=\left(H^{1}(\Omega)\right)^{2}$. Thus, the operators $L \in \mathcal{L}(\mathfrak{U}), M \in \mathcal{C l}(\mathfrak{U})$, and $N \in \mathcal{C l}(\mathfrak{U})$ are well defined, and $\operatorname{ker} L=\{0\} \times L_{2}(\Omega)$.

Denote $A w=\Delta w, \operatorname{dom} A=H_{\frac{\partial}{\partial n}+\lambda}^{2}(\Omega) \subset L_{2}(\Omega)$. Let $\left\{\varphi_{k}: k \in \mathbb{N}\right\}$ be the orthonormal eigenfunctions with respect to the inner product $\langle\cdot, \cdot\rangle$ in $L_{2}(\Omega)$ of the operator $A$, enumerated in the nonascending order of its eigenvalues $\left\{\lambda_{k}: k \in \mathbb{N}\right\}$, with regard to multiplicity.

Theorem 6.1 [21]. Suppose $-\beta \notin \sigma(A)$. Then the operator $M$ is strongly $(L, 0)$-radial.

In [21], the semigroup of the nonperturbed system (6.2)-(6.4),

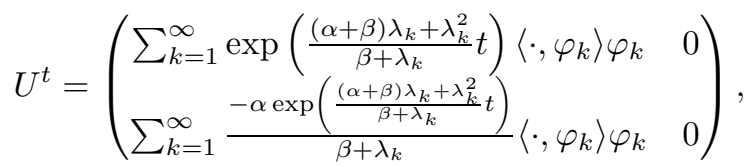

and the projections

$$
P=\left(\begin{array}{cc}
I & 0 \\
-\alpha(\beta+A)^{-1} & 0
\end{array}\right), \quad Q=\left(\begin{array}{cc}
I & A(\beta+A)^{-1} \\
0 & 0
\end{array}\right)
$$

were also obtained. Therefore, $\mathfrak{U}^{1}=\operatorname{im} P=\left\{\left(u,-\alpha(\beta+A)^{-1} u\right) \in\left(L_{2}(\Omega)\right)^{2}: u \in L_{2}(\Omega)\right\}$ is isomorphic to $L_{2}(\Omega) \times\{0\}$, and $\mathfrak{F}^{1}=\operatorname{im} Q=\left\{\left(u+A(\beta+A)^{-1} v, 0\right) \in\left(L_{2}(\Omega)\right)^{2}:(u, v) \in\right.$ $\left.\left(L_{2}(\Omega)\right)^{2}\right\}=L_{2}(\Omega) \times\{0\}$. Consequently, (6.1) is the generalized Showalter condition for this system of equations.

Lemma 6.1. Under the conditions of this section,

$$
N_{2}=\left(\begin{array}{cc}
a_{0}(x)+\sum_{i=1}^{s} a_{i}(x) \frac{\partial}{\partial x_{i}} & b_{0}(x)+\sum_{j=1}^{s} b_{j}(x) \frac{\partial}{\partial x_{j}} \\
0 & 0
\end{array}\right) \in \mathfrak{P}(L, M) .
$$

Proof. We have $\operatorname{dom} M \subset \operatorname{dom} N_{2}$, and

$$
\begin{aligned}
& N_{2} U^{t}\left(\begin{array}{l}
u \\
v
\end{array}\right)=N_{2}\left(\begin{array}{c}
\sum_{k=1}^{\infty} \exp \left(\frac{(\alpha+\beta) \lambda_{k}+\lambda_{k}^{2}}{\beta+\lambda_{k}} t\right)\left\langle u, \varphi_{k}\right\rangle \varphi_{k} \\
\sum_{k=1}^{\infty} \frac{-\alpha \exp \left(\frac{(\alpha+\beta) \lambda_{k}+\lambda_{k}^{2}}{\beta+\lambda_{k}} t\right)}{\beta+\lambda_{k}}\left\langle u, \varphi_{k}\right\rangle \varphi_{k}
\end{array}\right) \\
& =\left(\begin{array}{c}
\left(a_{0}+\sum_{i=1}^{s} a_{i} \frac{\partial}{\partial x_{i}}\right) \sum_{k=1}^{\infty} e^{\mu_{k} t}\left\langle u, \varphi_{k}\right\rangle \varphi_{k}+\left(b_{0}+\sum_{j=1}^{s} b_{j} \frac{\partial}{\partial x_{j}}\right) \sum_{k=1}^{\infty} \frac{-\alpha e^{\mu_{k} t}}{\beta+\lambda_{k}}\left\langle u, \varphi_{k}\right\rangle \varphi_{k} \\
0
\end{array}\right),
\end{aligned}
$$

where $\mu_{k}=\frac{(\alpha+\beta) \lambda_{k}+\lambda_{k}^{2}}{\beta+\lambda_{k}}$. Moreover,

$$
\begin{aligned}
\left(b_{0}(x)+\sum_{j=1}^{s} b_{j}(x) \frac{\partial}{\partial x_{j}}\right) & \sum_{k=1}^{\infty} \frac{-\alpha e^{\mu_{k} t}}{\beta+\lambda_{k}}\left\langle u, \varphi_{k}\right\rangle \varphi_{k} \\
& =-\alpha\left(b_{0}(x)+\sum_{j=1}^{s} b_{j}(x) \frac{\partial}{\partial x_{j}}\right)(\beta+A)^{-1} \sum_{k=1}^{\infty} e^{\mu_{k} t}\left\langle u, \varphi_{k}\right\rangle \varphi_{k} .
\end{aligned}
$$

Denoting $a=\max _{k \in \mathbb{N}} \mu_{k}$, for $u \in L_{2}(\Omega)$ we have

$$
\begin{aligned}
& \left\|\sum_{k=1}^{\infty} e^{\mu_{k} t}\left\langle u, \varphi_{k}\right\rangle \varphi_{k}\right\|_{L_{2}(\Omega)} \leq e^{a t}\|u\|_{L_{2}(\Omega)}, \\
& \left\|\left(b_{0}(x)+\sum_{j=1}^{s} b_{j}(x) \frac{\partial}{\partial x_{j}}\right)(\beta+A)^{-1} u\right\|_{L_{2}(\Omega)} \\
& \quad \leq \sup _{j=0, \ldots, s}\left\|b_{j}\right\|_{L_{\infty}(\Omega)}\left\|(\beta+A)^{-1} u\right\|_{H^{1}(\Omega)} \leq c_{1}\left\|(\beta+A)^{-1} u\right\|_{H^{2}(\Omega)} \leq c\|u\|_{L_{2}(\Omega)} .
\end{aligned}
$$


We have used the fact that $-\beta \in \rho(A)$, so that the operator $(\beta+A)^{-1}$ is a one-to-one continuous map from $L_{2}(\Omega)$ into $H_{\frac{\partial}{\partial n}+\lambda}^{2}(\Omega)$. Thus,

$$
\left\|\left(b_{0}(x)+\sum_{j=1}^{s} b_{j}(x) \frac{\partial}{\partial x_{j}}\right) \sum_{k=1}^{\infty} \frac{-\alpha e^{\mu_{k} t}}{\beta+\lambda_{k}}\left\langle u, \varphi_{k}\right\rangle \varphi_{k}\right\|_{L_{2}(\Omega)} \leq C\|u\|_{L_{2}(\Omega)}
$$

for all $u$ in $L_{2}(\Omega)$. Next,

$$
\begin{aligned}
\left(a_{0}(x)\right. & \left.+\sum_{i=1}^{s} a_{i}(x) \frac{\partial}{\partial x_{i}}\right) \sum_{k=1}^{\infty} e^{\mu_{k} t}\left\langle u, \varphi_{k}\right\rangle \varphi_{k} \\
& =\left(a_{0}(x)+\sum_{i=1}^{s} a_{i}(x) \frac{\partial}{\partial x_{i}}\right)(b+1-A)^{-\frac{2}{3}} \sum_{k=1}^{\infty}\left(\frac{b+1-\lambda_{k}}{\mu_{k}}\right)^{\frac{2}{3}} \mu_{k}^{\frac{2}{3}} e^{\mu_{k} t}\left\langle u, \varphi_{k}\right\rangle \varphi_{k},
\end{aligned}
$$

where $b=\max _{k \in \mathbb{N}} \lambda_{k}$. We argue as in the proof of Lemma 5.2. The operator $A$ is sectorial and we have a continuous and dense embedding of the Banach space $\operatorname{dom}(b+1-A)^{\frac{2}{3}}$ in $H^{1}(\Omega)$. Consequently, for $u \in L_{2}(\Omega)$ we have

$$
\begin{aligned}
& \left\|\left(a_{0}(x)+\sum_{i=1}^{s} a_{i}(x) \frac{\partial}{\partial x_{i}}\right)(b+1-A)^{-\frac{2}{3}} u\right\|_{L_{2}(\Omega)} \\
& \quad \leq \sup _{i=0, \ldots, s}\left\|a_{i}\right\|_{L_{\infty}(\Omega)}\left\|(b+1-A)^{-\frac{2}{3}} u\right\|_{H^{1}(\Omega)} \leq c\left\|(b+1-A)^{-\frac{2}{3}} u\right\|_{\frac{2}{3}}=c\|u\|_{L_{2}(\Omega)}, \\
& \left\|\sum_{k=1}^{\infty}\left(\frac{a+1+\lambda_{k}}{\mu_{k}}\right)^{\frac{2}{3}} \mu_{k}^{\frac{2}{3}} e^{\mu_{k} t}\left\langle u, \varphi_{k}\right\rangle \varphi_{k}\right\|_{L_{2}(\Omega)} \\
& \quad \leq \max _{k \in \mathbb{N}}\left(\frac{a+1+\lambda_{k}}{\mu_{k}}\right)^{\frac{2}{3}}\left\|\sum_{k=1}^{\infty} \mu_{k}^{\frac{2}{3}} e^{\mu_{k} t}\left\langle u, \varphi_{k}\right\rangle \varphi_{k}\right\|_{L_{2}(\Omega)} \leq c t^{-\frac{2}{3}}\|u\|_{L_{2}(\Omega)} .
\end{aligned}
$$

This proves that $\left\|N_{2} U^{t}\right\|_{\mathcal{L}\left(L_{2}(\Omega)\right)} \leq c t^{-\frac{2}{3}}$, as required.

Theorem 6.2. Suppose $-\beta \notin \sigma(A), u_{0} \in H_{\frac{\partial}{\partial n}+\lambda}^{2}(\Omega)$. Then problem (6.1)-(6.4) has a unique solution $(u, v) \in\left(C^{1}\left(\overline{\mathbb{R}}_{+} ; L_{2}(\Omega)\right)\right)^{2}$.

Proof. Obviously, $\operatorname{im} N \subset \mathfrak{F}^{1}$. By Lemma 6.1 and Remarks 3.1, 3.2, we have $N=$ $N_{1}+N_{2} \in \mathfrak{P}(L, M)$. It remains to refer to Theorem 3.4.

Consider the system

$$
\begin{aligned}
u_{t}(x, t)= & \Delta u(x, t)-\Delta v(x, t) \\
& +\int_{\Omega} K_{1}(x, y) u(y, t) d y+a_{0}(x) u+\sum_{i=1}^{s} a_{i}(x) \frac{\partial u}{\partial x_{i}}, \quad(x, t) \in \Omega \times \overline{\mathbb{R}}_{+},
\end{aligned}
$$

$$
\begin{aligned}
\Delta v(x, t) & +\beta v(x, t)+\alpha u(x, t) \\
& +\int_{\Omega} K_{2}(x, y) u(y, t) d y+b_{0}(x) u+\sum_{j=1}^{s} b_{j}(x) \frac{\partial u}{\partial x_{j}}=0, \quad(x, t) \in \Omega \times \overline{\mathbb{R}}_{+} .
\end{aligned}
$$

As before, $K_{m} \in L_{2}(\Omega \times \Omega), m=1,2, a_{i}, b_{j} \in L_{\infty}(\Omega), i, j=0, \ldots, s$. In this case,

$$
N_{1}\left(\begin{array}{l}
u \\
v
\end{array}\right)=\left(\begin{array}{l}
\int_{\Omega} K_{1}(x, y) u(y) d y \\
\int_{\Omega} K_{2}(x, y) u(y) d y
\end{array}\right), \quad N_{2}=\left(\begin{array}{ll}
a_{0}(x)+\sum_{i=1}^{s} a_{i}(x) \frac{\partial}{\partial x_{i}} & 0 \\
b_{0}(x)+\sum_{j=1}^{s} b_{j}(x) \frac{\partial}{\partial x_{j}} & 0
\end{array}\right),
$$

$N_{1} \in \mathcal{L}(\mathfrak{U}), N=N_{1}+N_{2}, \operatorname{dom} N=\operatorname{dom} N_{2}=H^{1}(\Omega) \times L_{2}(\Omega), N_{2}, N \in \mathcal{C l}(\mathfrak{U})$. 
Lemma 6.2. Under the conditions of this section,

$$
N_{2}=\left(\begin{array}{ll}
a_{0}(x)+\sum_{i=1}^{s} a_{i}(x) \frac{\partial}{\partial x_{i}} & 0 \\
b_{0}(x)+\sum_{j=1}^{s} b_{j}(x) \frac{\partial}{\partial x_{j}} & 0
\end{array}\right) \in \mathfrak{P}(L, M) .
$$

Proof. The embedding $\operatorname{dom} M \subset \operatorname{dom} N_{2}$ is obvious. Next, we have

$$
N_{2} U^{t}\left(\begin{array}{l}
u \\
v
\end{array}\right)=\left(\begin{array}{l}
\left(a_{0}(x)+\sum_{i=1}^{s} a_{i}(x) \frac{\partial}{\partial x_{i}}\right) \sum_{k=1}^{\infty} e^{\mu_{k} t}\left\langle u, \varphi_{k}\right\rangle \varphi_{k} \\
\left(b_{0}(x)+\sum_{j=1}^{s} b_{j}(x) \frac{\partial}{\partial x_{j}}\right) \sum_{k=1}^{\infty} e^{\mu_{k} t}\left\langle u, \varphi_{k}\right\rangle \varphi_{k}
\end{array}\right) .
$$

Therefore, as under the conditions of Lemma 6.1, the estimate

$$
\left\|N_{2} U^{t}\right\|_{\mathcal{L}\left(L_{2}(\Omega)\right)} \leq c t^{-\frac{2}{3}}
$$

is fulfilled.

Theorem 6.3. Suppose $-\beta \notin \sigma(A), u_{0} \in H_{\frac{\partial}{\partial n}+\lambda}^{2}(\Omega)$. Then problem (6.1), (6.2), (6.5), (6.6) has a unique solution $(u, v) \in\left(C^{1}\left(\overline{\mathbb{R}}_{+} ; L_{2}(\Omega)\right)\right)^{2}$.

Proof. As in the proof of Theorem 6.2, we obtain $N=N_{1}+N_{2} \in \mathfrak{P}(L, M)$. Moreover, $\mathfrak{U}^{0}=\operatorname{ker} P=\{0\} \times L_{2}(\Omega) \subset \operatorname{ker} N$. It remains to use Theorem 3.5 and Lemma 5.1.

\section{§7. Linearized NAVIER-STOKES System}

Consider the initial boundary value problem

$$
\begin{aligned}
& u(x, 0)=u_{0}(x), \quad x \in \Omega, \\
& u(x, t)=0, \quad(x, t) \in \partial \Omega \times \overline{\mathbb{R}}_{+},
\end{aligned}
$$

for the linearized Navier-Stokes system with integral perturbation

$$
\begin{aligned}
u_{t}(x, t)= & \nu \Delta u(x, t)-r(x, t) \\
& +\int_{\Omega} K_{1}(x, y) u(y, t) d y+\int_{\Omega} K_{2}(x, y) r(y, t) d y, \quad(x, t) \in \Omega \times \overline{\mathbb{R}}_{+}, \\
\nabla \cdot u= & 0, \quad(x, t) \in \Omega \times \overline{\mathbb{R}}_{+} .
\end{aligned}
$$

Here $\nu>0, \Omega \subset \mathbb{R}^{n}$ is a bounded domain with boundary $\partial \Omega$ of class $C^{\infty}$, and $r=$ $r(x, t)=\nabla p$ is the pressure gradient.

We denote $\mathbb{H}^{2}=\left(H^{2}(\Omega)\right)^{n}, \mathbb{H}_{0}^{2}=\left\{w \in \mathbb{H}^{2}: w(x)=0, x \in \partial \Omega\right\}, \mathbb{L}_{2}=\left(L_{2}(\Omega)\right)^{n}$, $\mathcal{L}=\left\{w \in\left(C_{0}^{\infty}(\Omega)\right)^{n}: \nabla \cdot w=0\right\}$. The closure of the subspace $\mathcal{L}$ with respect the norm of the space $\mathbb{L}_{2}$ will be denoted by $\mathbb{H}_{\sigma}$. This is a Hilbert space with the inner product of the space $\mathbb{L}_{2}$. We can decompose $\mathbb{L}_{2}=\mathbb{H}_{\sigma} \oplus \mathbb{H}_{\pi}$, where $\mathbb{H}_{\pi}$ is the orthogonal complement of $\mathbb{H}_{\sigma}$. Let $\Pi: \mathbb{L}_{2} \rightarrow \mathbb{H}_{\pi}$ denote the orthogonal projection corresponding to this decomposition. The restriction of $\Pi$ to the space $\mathbb{H}_{0}^{2} \subset \mathbb{L}_{2}$ is a continuous operator $\Pi_{1}: \mathbb{H}_{0}^{2} \rightarrow \mathbb{H}_{0}^{2}$. Therefore, $\mathbb{H}_{0}^{2}$ is the direct sum $\mathbb{H}_{\sigma}^{2} \oplus \mathbb{H}_{\pi}^{2}$, where $\mathbb{H}_{\sigma}^{2}=\operatorname{ker} \Pi_{1}$ and $\mathbb{H}_{\pi}^{2}=\mathrm{im} \Pi_{1}$.

We replace the incompressibility equation (7.4) with a more general equation:

$$
\Pi u(x, t)=0, \quad(x, t) \in \Omega \times \overline{\mathbb{R}}_{+} .
$$

Indeed, if $u(x)$ is sufficiently smooth, then $\Pi u \equiv 0$ implies (7.4). Otherwise, by (7.5), $u$ is the limit in $\mathbb{L}_{2}$ of smooth functions satisfying condition (7.4).

It is easy to observe that the formula $A=\operatorname{diag}\{\Delta, \ldots, \Delta\}$ determines a continuous linear operator $A: \mathbb{H}_{0}^{2} \rightarrow \mathbb{L}_{2}$ with discrete spectrum $\sigma(A)$; this spectrum has finite multiplicity and condenses only at $-\infty$.

Like $\mathbb{H}_{0}^{2}$, the space $\mathbb{H}_{0, A}^{4}=\left\{w \in \mathbb{H}^{4}: w(x)=A w(x)=0, x \in \partial \Omega\right\}$ can be decomposed as $\mathbb{H}_{\sigma}^{4} \oplus \mathbb{H}_{\pi}^{4}$. 
We denote $A_{\sigma}=\left.A\right|_{\mathbb{H}_{\sigma}^{2}}, A_{\pi}=\left.A\right|_{\mathbb{H}_{\pi}^{2}}, \Sigma=I-\Pi$.

Lemma 7.1. $A_{\sigma}$ acts from $\mathbb{H}_{\sigma}^{2}$ to $\mathbb{H}_{\sigma}$, and $A_{\pi}$ acts from $\mathbb{H}_{\pi}^{2}$ to $\mathbb{H}_{\pi}$.

Proof. We show that $\Pi A \Sigma \equiv \mathbb{O}$. Indeed, for $v \in \mathcal{L}$ we have $(\nabla \cdot A v)=\Delta(\nabla \cdot v)=0$. This relation extends to $\mathbb{H}_{\sigma}^{2}$ by continuity. Hence, $A_{\sigma}: \mathbb{H}_{\sigma}^{2} \rightarrow \mathbb{H}_{\sigma}$.

Next we show that $\Sigma A \Pi \equiv \mathbb{O}$. Take $v \in \mathbb{H}_{\sigma}^{2}, u \in \mathbb{H}_{\pi}^{2}$. Let $\langle\cdot, \cdot\rangle$ be the inner product in $\mathbb{L}_{2}$. We have $\langle v, A u\rangle=\langle A v, u\rangle=0$, because $A$ is selfadjoint, $A v \in \mathbb{H}_{\sigma}$ (see above), and the spaces $\mathbb{H}_{\sigma}$ and $\mathbb{H}_{\pi}$ are mutually orthogonal. Since $\mathbb{H}_{\sigma}^{2}$ is dense in $\mathbb{H}_{\sigma}$, we have $A u \perp \mathbb{H}_{\sigma}$, so that $A_{\pi}: \mathbb{H}_{\pi}^{2} \rightarrow \mathbb{H}_{\pi}$.

Put $\mathfrak{U}=\mathfrak{F}=\mathbb{H}_{\sigma} \times \mathbb{H}_{\pi} \times \mathbb{H}_{r}, \mathbb{H}_{r}=\mathbb{H}_{\pi}$. Then for vectors $v \in \mathfrak{U}, f \in \mathfrak{F}$ we have $v=\left(v_{\sigma}, v_{\pi}, v_{r}\right), f=\left(f_{\sigma}, f_{\pi}, f_{r}\right)$, and

$$
L=\left(\begin{array}{lll}
1 & 0 & 0 \\
0 & 1 & 0 \\
0 & 0 & 0
\end{array}\right), \quad M=\left(\begin{array}{ccc}
\nu A_{\sigma} & 0 & 0 \\
0 & \nu A_{\pi} & -1 \\
0 & -1 & 0
\end{array}\right)
$$

Then $L: \mathfrak{U} \rightarrow \mathfrak{F}$ is a continuous linear operator with ker $L=\{0\} \times\{0\} \times \mathbb{H}_{r}$ and $\operatorname{im} L=\mathbb{H}_{\sigma} \times \mathbb{H}_{\pi} \times\{0\}$. The domain of the closed and densely defined operator $M: \mathfrak{U} \rightarrow \mathfrak{F}$ is $\operatorname{dom} M=\mathbb{H}_{\sigma}^{2} \times \mathbb{H}_{\pi}^{2} \times \mathbb{H}_{r}$.

Theorem 7.1. The operator $M$ is strongly $(L, 1)$-radial.

Proof. We have

$$
\mu L-M=\left(\begin{array}{ccc}
\mu-\nu A_{\sigma} & 0 & 0 \\
0 & \mu-\nu A_{\pi} & 1 \\
0 & 1 & 0
\end{array}\right) .
$$

By Lemma 7.1, the operator $A_{\sigma}: \mathbb{H}_{\sigma}^{2} \rightarrow \mathbb{H}_{\sigma}$ is continuous and linear, and its spectrum $\sigma\left(A_{\sigma}\right)$ is discrete, has finite multiplicity and condenses only at $-\infty$, because the subspace $\mathbb{H}_{\sigma}$ is infinite-dimensional. Let $\left\{\varphi_{k}\right\}$ be the eigenfunctions of $A_{\sigma}$ orthonormal in $\mathbb{H}_{\sigma}$, enumerated in the nonascending order of the corresponding eigenvalues $\left\{\lambda_{k}: k \in \mathbb{N}\right\}$, with regard to multiplicity. Then $\Sigma(\mu-\nu A) \Sigma=\left(\mu-\nu A_{\sigma}\right) \Sigma=\sum_{k=1}^{\infty}\left(\mu-\nu \lambda_{k}\right)\left\langle\cdot, \varphi_{k}\right\rangle \varphi_{k}$, where $\langle\cdot, \cdot\rangle$ is the inner product in $\mathbb{L}_{2}$. If $\mu \neq \nu \lambda_{k}$, then we have a continuous operator

$$
A_{\sigma \mu}^{-1} \equiv\left(\mu-\nu A_{\sigma}\right)^{-1}=\sum_{k=1}^{\infty} \frac{\left\langle\cdot, \varphi_{k}\right\rangle \varphi_{k}}{\mu-\nu \lambda_{k}}: \mathbb{H}_{\sigma} \rightarrow \mathbb{H}_{\sigma}
$$

For $\mu \neq \nu \lambda_{k}$ we can construct the $L$-resolvent of $M$ :

$$
(\mu L-M)^{-1}=\left(\begin{array}{ccc}
A_{\sigma \mu}^{-1} & 0 & 0 \\
0 & 0 & 1 \\
0 & 1 & -\left(\mu-\nu A_{\pi}\right)
\end{array}\right) .
$$


Thus,

$$
\begin{aligned}
& R_{\mu}^{L}(M)=\left(\begin{array}{ccc}
A_{\sigma \mu}^{-1} & 0 & 0 \\
0 & 0 & 0 \\
0 & 1 & 0
\end{array}\right), R_{(\mu, 1)}^{L}(M)=\left(\begin{array}{ccc}
A_{\sigma \mu_{0}}^{-1} A_{\sigma \mu_{1}}^{-1} & 0 & 0 \\
0 & 0 & 0 \\
0 & 0 & 0
\end{array}\right), \\
& L_{\mu}^{L}(M)=\left(\begin{array}{ccc}
A_{\sigma \mu}^{-1} & 0 & 0 \\
0 & 0 & 1 \\
0 & 0 & 0
\end{array}\right), \quad L_{(\mu, 1)}^{L}(M)=\left(\begin{array}{ccc}
A_{\sigma \mu_{0}}^{-1} A_{\sigma \mu_{1}}^{-1} & 0 & 0 \\
0 & 0 & 0 \\
0 & 0 & 0
\end{array}\right), \\
& R_{(\mu, 1)}^{L}(M)(\lambda L-M)^{-1}=\left(\begin{array}{ccc}
A_{\sigma \mu_{0}}^{-1} A_{\sigma \mu_{1}}^{-1} A_{\sigma \lambda}^{-1} & 0 & 0 \\
0 & 0 & 0 \\
0 & 0 & 0
\end{array}\right), \\
& M(\lambda L-M)^{-1} L_{(\mu, 1)}^{L}(M) f=\left(\begin{array}{ccc}
\nu A_{\sigma \mu_{0}}^{-1} A_{\sigma \mu_{1}}^{-1} A_{\sigma \lambda}^{-1} A f_{\sigma} & 0 & 0 \\
0 & 0 & 0 \\
0 & 0 & 0
\end{array}\right),
\end{aligned}
$$

where $f \in \stackrel{\circ}{\mathfrak{F}}=\mathbb{H}_{\sigma}^{2} \times \mathbb{H}_{\pi} \times \mathbb{H}_{r}$. The obvious inequality $\left\|A_{\sigma \mu}^{-1}\right\|_{\mathcal{L}\left(\mathbb{H}_{\sigma}\right)} \leq\left(\mu-\nu \lambda_{1}\right)^{-1}$ for $\mu>\nu \lambda_{1}$ implies that $M$ is strongly $(L, 1)$-radial.

Since, by Remark 2.1,

$$
P=s-\lim _{\mu \rightarrow+\infty}\left(\mu R_{\mu}^{L}(M)\right)^{2}, \quad Q=\underset{\mu-\lim }{s \rightarrow+\infty}\left(\mu L_{\mu}^{L}(M)\right)^{2},
$$

it is easy to check that

$$
P=Q=\left(\begin{array}{lll}
1 & 0 & 0 \\
0 & 0 & 0 \\
0 & 0 & 0
\end{array}\right)
$$

Theorem 7.2. Let $u_{0} \in \mathbb{H}_{\sigma}^{2}$, and let $K_{i}(\cdot, y) \in \mathbb{H}_{\sigma}$ for almost all $y \in \Omega, i=1,2$. Then problem (7.1)-(7.3), (7.5) has a unique solution $u \in C^{1}\left(\overline{\mathbb{R}}_{+} ; \mathfrak{U}\right)$.

Proof. The initial value $u_{0}$ of the problem belongs to the set $\operatorname{dom} M_{1}=\operatorname{dom} M \cap \operatorname{im} P=$ $\mathbb{H}_{\sigma}^{2} \times\{0\} \times\{0\}$. Take $v=(\Sigma u, \Pi u, r)$. Since $\mathfrak{F}^{1}=\operatorname{im} Q=\mathbb{H}_{\sigma} \times\{0\} \times\{0\}$, we have

$$
N v=\left(\begin{array}{c}
\Sigma \int_{\Omega} K_{1}(x, y) u(y) d y+\Sigma \int_{\Omega} K_{2}(x, y) r(y) d y \\
\Pi \int_{\Omega} K_{1}(x, y) u(y) d y+\Pi \int_{\Omega} K_{2}(x, y) r(y) d y \\
0
\end{array}\right) \in \mathfrak{F}^{1}
$$

by the assumptions of the theorem concerning the functions $K_{i}, i=1,2$. We have used the continuity of the operator $\Pi$. It remains to refer to Theorem 3.4.

Now we consider a similar system with integral perturbation of another form:

$$
\begin{aligned}
u_{t}(x, t) & =\nu \Delta u(x, t)-r(x, t)+\int_{\Omega} K_{1}(x, y) u(y, t) d y, \quad(x, t) \in \Omega \times \overline{\mathbb{R}}_{+}, \\
\Pi u & =\int_{\Omega} K_{2}(x, y) u(y, t) d y, \quad(x, t) \in \Omega \times \overline{\mathbb{R}}_{+} .
\end{aligned}
$$

Theorem 7.3. Suppose $u_{0} \in \mathbb{H}_{\sigma}^{4}, K_{i}(x, \cdot) \in \mathbb{H}_{\sigma}$ for almost all $x \in \Omega, i=1,2$, and $K_{2}(\cdot, y) \in \mathbb{H}_{\pi}$ for almost all $y \in \Omega$. Then problem (7.1), (7.2), (7.6), (7.7) has a unique solution $u \in C^{1}\left(\overline{\mathbb{R}}_{+} ; \mathfrak{U}\right)$.

Proof. We have $\mathfrak{U}^{0}=\operatorname{ker} P=\{0\} \times \mathbb{H}_{\pi} \times \mathbb{H}_{r}$ and, for $v=\left(u_{\sigma}, u_{\pi}, r\right)$,

$$
N v=\left(\begin{array}{c}
\Sigma \int_{\Omega} K_{1}(x, y) u_{\sigma}(y) d y+\Sigma \int_{\Omega} K_{1}(x, y) u_{\pi}(y) d y \\
\Pi \int_{\Omega} K_{1}(x, y) u_{\sigma}(y) d y+\Pi \int_{\Omega} K_{1}(x, y) u_{\pi}(y) d y \\
\int_{\Omega} K_{2}(x, y) u_{\sigma}(y) d y+\int_{\Omega} K_{2}(x, y) u_{\pi}(y) d y
\end{array}\right) .
$$


Therefore, the conditions imposed on the functions $K_{i}(x, \cdot), i=1,2$, for fixed $x \in \Omega$ imply the condition $\mathfrak{U}^{0} \subset \operatorname{ker} N$ of Theorem 3.5. The condition imposed on the functions $K_{2}(\cdot, y)$ for fixed $y \in \Omega$ is necessary for the solvability of equation (7.7), because (7.7) implies the relations

$$
\Pi \int_{\Omega} K_{2}(x, y) u(y, t) d y=\Pi^{2} u=\Pi u=\int_{\Omega} K_{2}(x, y) u(y, t) d y, \quad(x, t) \in \Omega \times \overline{\mathbb{R}}_{+} .
$$

Therefore, $\Pi K_{2}(\cdot, y)=K_{2}(\cdot, y)$ for almost all $y \in \Omega$. It remains to observe that $u_{0} \in$ $\operatorname{im} R_{(\mu, 1)}^{L}(M)$ if and only if $u_{0} \in \operatorname{dom} A_{\sigma}^{2}$, which means that $u_{0} \in \mathbb{H}_{\sigma}^{4}$. Thus, all the assumptions of Theorem 3.5 are fulfilled.

\section{REFERENCES}

[1] G. A. Sviridyuk, On the general theory of operator semigroups, Uspekhi Mat. Nauk 49 (1994), no. 4, 47-74; English transl., Russian Math. Surveys 49 (1994), no. 4, 45-74. MR.1309441 (96i:34135)

[2] G. V. Demidenko and S. V. Uspenskiı̌, Partial differential equations and systems not solvable with respect to the highest-order derivative, Nauchnaya Kniga, Novosibirsk, 1998; English transl., Monogr. Textbooks Pure Appl. Math., vol. 256, Marcel Dekker, Inc., New York, 2003. MR1831690 (2002j:35001) MR2064818 (2005a:35001)

[3] G. A. Sviridyuk and V. E. Fedorov, Linear Sobolev type equations and degenerate semigroups of operators, VSP, Utrecht, 2003. MR2225515 (2007c:47095)

[4] A. G. Sveshnikov, A. B. Al'shin, M. O. Korpusov, and Yu. D. Pletner, Linear and nonlinear equations of Sobolev type, Fizmatlit, Moscow, 2007. (Russian)

[5] A. Favini and A. Yagi, Degenerate differential equations in Banach spaces, Monogr. Textbooks Pure Appl. Math., vol. 215, Marcel Dekker, Inc., New York, 1999. MR.1654663 (99i:34079)

[6] V. E. Fedorov, Degenerate strongly continuous semigroups of operators, Algebra i Analiz 12 (2000), no. 3, 173-200; English transl., St. Petersburg Math. J. 12 (2001), no. 3, 471-489. MR1778194 (2001k:47060)

[7] Holomorphic resolving semigroups of Sobolev-type equations in locally convex spaces, Mat. Sb. 195 (2004), no. 8, 131-160; English transl., Sb. Math. 195 (2004), no. 7-8, 1205-1234. MR 2101340 (2005i:47065)

[8] _ A generalization of the Hille-Yosida theorem to the case of degenerate semigroups in locally convex spaces, Sibirsk. Mat. Zh. 46 (2005), no. 2, 426-448; English transl., Siberian Math. J. 46 (2005), no. 2, 333-350. MR2141208 (2006c:47045)

[9] R. S. Phillips, Perturbation theory for semi-groups of linear operators, Trans. Amer. Math. Soc. 74 (1953), 199-221. MR0054167(14:882b)

[10] E. Hille and R. S. Phillips, Functional analysis and semi-groups, Amer. Math. Soc. Colloq. Publ., vol. 31, Amer. Math. Soc., Providence, RI, 1957. MR0089373 (19:664d)

[11] S. G. Krĕn and M. I. Khazan, Differential equations in Banach space, Itogi Nauki i Tekhniki. Ser. Mat. Anal., vol. 21, VINITI, Moscow, 1983, pp. 130-264; English transl. in J. Soviet Math. 30 (1985), no. 3. MR0736523 (85f:34116)

[12] V. V. Ivanov, Perturbation of uniformly summable operator semigroups, Dokl. Akad. Nauk SSSR 250 (1980), no. 2, 269-273; English transl., Soviet Math. Dokl. 21 (1980), no. 1, 48-52. MR0557767 (81d:47030)

[13] , Uniformly summable semigroups of operators. II. Perturbation of the semigroups, Trudy Inst. Mat. (Novosibirsk), vol. 9, Nauka, Novosibirsk, 1987, pp. 159-182. (Russian) MR0929254 (89d:47090)

[14] N. Dunford and J. T. Schwartz, Linear operators. Part I. General theory, John Wiley and Sons, Inc., New York, 1988. MR1009162(90g:47001a)

[15] G. A. Sviridyuk and N. A. Manakova, Regular perturbations of a class of linear equations of Sobolev type, Differ. Uravn. 38 (2002), no. 3, 423-425; English transl., Differ. Equ. 38 (2002), no. 3, 447-450. MR.2005085 (2004f:34088)

[16] H. Triebel, Interpolation theory, function spaces, differential operators, 2nd ed., Johann Ambrosius Barth, Heidelberg, 1995. MR1328645 (96f:46001)

[17] R. E. Showalter, Existence and representation theorems for a semilinear Sobolev equation in Banach space, SIAM J. Math. Anal. 3 (1972), no. 3, 527-543. MR0315239 (47:3788)

[18] D. Henry, Geometric theory of semilinear parabolic equations, Lecture Notes in Math., vol. 840, Springer-Verlag, Berlin-New York, 1981. MR0610244 (83j:35084) 
[19] P. I. Plotnikov and V. N. Starovoltov, The Stefan problem with surface tension as a limit of the phase field model, Differ. Uravn. 29 (1993), no. 3, 461-471; English transl., Differ. Equ. 29 (1993), no. 3, 395-404. MR1236334 (94f:35155)

[20] P. I. Plotnikov and A. V. Klepacheva, Phase field equations and gradient flows of marginal functions, Sibirsk. Mat. Zh. 42 (2001), no. 3, 651-669; English transl., Siberian Math. J. 42 (2001), no. 3, 551-567. MR.1852242 (2002e:35116)

[21] V. E. Fedorov and A. V. Urazaeva, An inverse problem for a class of singular linear operatordifferential equations, Trudy Voronezh. Zimneı̆ Mat. Shkoly, Voronezh. Gos. Univ., Voronezh, 2004, pp. 16-172. (Russian)

Chelyabinsk State University, Ul. Br. Kashirinykh, 454021 Chelyabinsk, Russia

E-mail address: kar@csu.ru

Chelyabinsk State University, Ul. Br. Kashirinykh, 454021 Chelyabinsk, Russia

E-mail address: amber@csu.ru

Received 14/APR/2007

Translated by THE AUTHORS 